\title{
HYDROGEOLOGY AND GROUND-WATER QUALITY IN THE BLACK BELT AREA OF WEST-CENTRAL ALABAMA, AND ESTIMATED WATER USE FOR AQUACULTURE, 1990
}

By Robert E. Kidd and Darrell S. Lambeth

U.S. GEOLOGICAL SURVEY

Water-Resources Investigations Report 94-4074

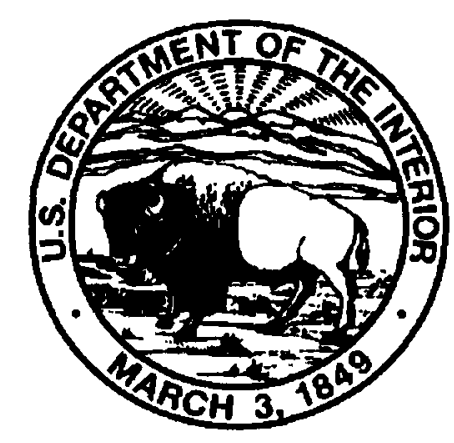

Prepared in cooperation with the

U.S. DEPARTMENT OF AGRICULTURE

SOIL AND CONSERVATION SERVICE and the ALABAMA DEPARTMENT OF

ECONOMIC AND COMMUNITY AFFAIRS

Tuscaloosa, Alabama

1995 


\title{
U.S. DEPARTMENT OF THE INTERIOR
}

BRUCE BABBITT, Secretary

\author{
U.S. GEOLOGICAL SURVEY
}

GORDON P. EATON, Director

For additional information write to:

\section{District Chief}

U.S. Geological Survey

520 19th Avenue

Tuscaloosa, Alabama 35401
Copies of this report can be purchased from:

U.S. Geological Survey

Earth Science Information Center

Open-File Reports Section

Box 25286, MS 517, Federal Center

Denver, Colorado 80225 


\section{CONTENTS}

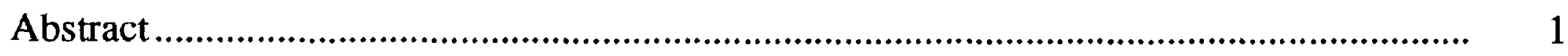

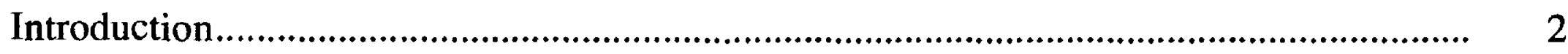

Description of the study area ................................................................................ 2

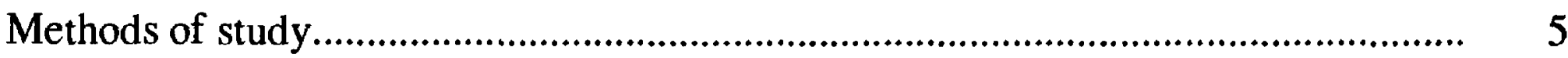

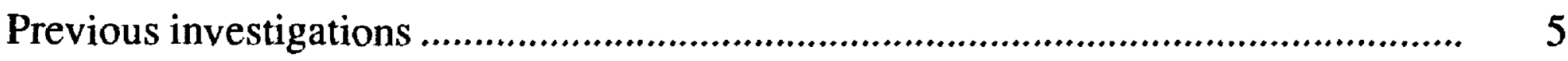

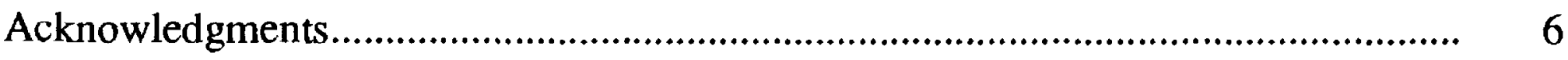

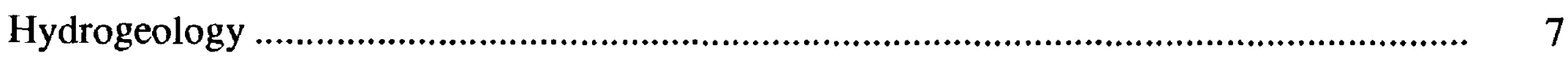

Deposits of Quaternary age................................................................................... 7

Deposits of Tertiary age .................................................................................. 22

Nanafalia Formation .................................................................................. 22

Naheola Formation ................................................................................. 22

Clayton and Porters Creek Formations .......................................................... 22

Deposits of Cretaceous age ................................................................................ 23

Prairie Bluff Chalk .................................................................................. 23

Ripley Formation....................................................................................... 23

Demopolis Chalk ......................................................................................... 24

Mooreville Chalk ............................................................................................ 24

Eutaw Formation .......................................................................................... 24

Gordo Formation ..................................................................................... 25

Coker Formation.......................................................................................... 25

Lower Cretaceous rocks ................................................................................. 26

Recharge to and discharge from the major aquifers.................................................... 26

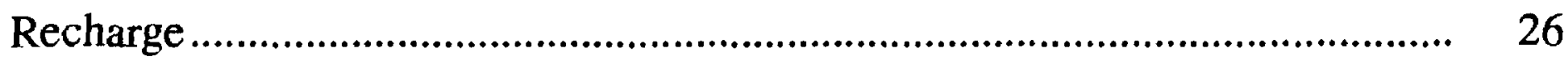

Natural discharge and ground-water withdrawals.............................................. 29

Ground-water quality .................................................................................................. 31

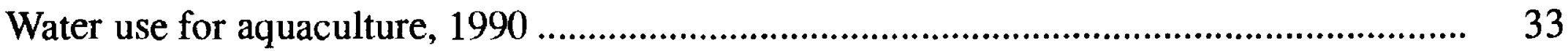

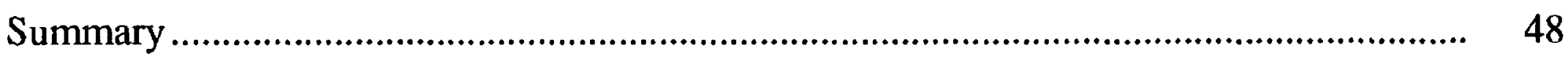

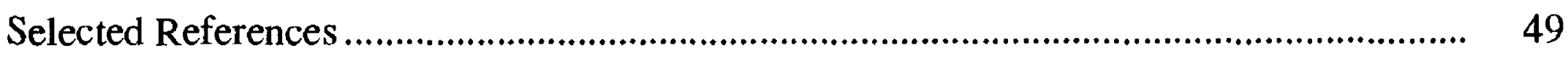




\section{ILLUSTRATIONS}

[Plates are in pocket]

Plate 1. Locations of catfish ponds in the Black Belt area of west-central Alabama, 1991

Plate 2. Locations of inventoried wells in the Black Belt area of west-central Alabama, 1991

Plate 3. Dissolved chloride concentrations of water from selected wells in the Black Belt area of west-central Alabama, March 12-21, 1991.

\section{FIGURES}

\section{1-4. Map showing:}

1. Location of the study area and selected continuous streamflow gaging stations...... 3

2. Physiographic districts of the Black Belt area of west-central Alabama ................... 4

3. Generalized geology of the Black Belt area of west-central Alabama ..................... 8

4. Estimated areas of recharge of the major aquifers in the Black Belt area of west-central Alabama................................................................................................ 9

5. Diagrammatic geologic section of the Black Belt area of west-central Alabama ........ $\quad 10$

6. Correlation chart showing geologic units and aquifers in the Black Belt area of west-central Alabama, and their water-bearing properties.................................... 11

7. Hydrographs of selected wells in the Eutaw aquifer in the Black Belt area of west-central Alabama ....................................................................................... 30

\section{TABLES}

1. Records of selected wells in the Black Belt area of west-central Alabama, 1991............. 12

2. Recharge to the major aquifers in the Black Belt area of west-central Alabama, estimated from baseflow of streams at selected gaging stations

3. Selected physical and chemical characteristics of water from wells in the Black Belt area of west-central Alabama, March 14-18, 1991

4. Records of selected catfish ponds in the Black Belt area of west-central Alabama, 1991

5. Estimated average annual water use for aquaculture in the Black Belt area of west-central Alabama in 1990, by county 


\section{CONVERSION FACTORS AND VERTICAL DATUM}

\section{Multiolv}

inch (in.)

foot $(\mathrm{ft})$

mile (mi)

square mile $\left(\mathrm{mi}^{2}\right)$

acre

gallon per minute (gal/min)

million gallons per day (Mgal/d)

inches per year (in/yr)

$\begin{array}{cl}\text { By } & \text { Toobtain } \\ 25.4 & \text { millimeter } \\ 0.3048 & \text { meter } \\ 1.609 & \text { kilometer } \\ 259.0 & \text { hectare } \\ 4,047 & \text { square meter } \\ 0.06308 & \text { liter per second } \\ 0.04381 & \text { cubic meter per second } \\ 2.54 & \text { centimeters per year }\end{array}$

Sea Level: In this report, "sea level" refers to the National Geodetic Vertical Datum of 1929--a geodetic datum derived from a general adjustment of the first-order level nets of the United States and Canada, formerly called Sea Level Datum of 1929. 


\title{
HYDROGEOLOGY AND GROUND-WATER QUALITY IN THE BLACK BELT AREA OF WEST-CENTRAL ALABAMA, AND ESTIMATED WATER USE FOR AQUACULTURE, 1990
}

\author{
By Robert E. Kidd and Darrell S. Lambeth
}

\section{ABSTRACT}

Commercial production of catfish in west-central Alabama began about 1970, and by 1991 catfish ponds covered about 16,000 acres in the Black Belt area of the State. The rapid increase in catfish farming or aquaculture and the associated demand for ground water led the U.S.

Geological Survey in cooperation with other Federal and State agencies in 1990 to initiate a study to better define the ground-water resources in the Black Belt area.

The major aquifers in the study area are sand and gravel beds in the Eutaw, Gordo, and Coker Formations. Recharge to these aquifers occurs primarily in areas where those formations crop out. The average recharge to the major aquifers in the study area, as estimated from baseflow analysis of streams in the outcrop area, is 11.4 inches per year.

Water from the major aquifers in the study area generally is of good quality and suitable for most uses. Water from the Eutaw aquifer, however, contains chloride in concentrations greater than 500 milligrams per liter in central Greene County and in downdip areas in Marengo and Wilcox Counties and is not suitable for public water supply. Some ground water with elevated chloride concentrations is used for catfish farming in these areas, however.

The total estimated water use for aquaculture in the study area in 1990 was 21.83 million gallons per day, 16.08 million gallons per day from ground-water sources, and 5.75 million gallons per day from surface water sources. About 13.54 million gallons per day of water was used for filling catfish ponds and an additional 8.29 million gallons per day was used to replace evaporation losses. 


\section{INTRODUCTION}

Commercial production of catfish in Alabama began about 1970 and by 1991 aquaculture ponds covered about 16,000 acres in and near the Black Belt area of west-central Alabama (plate 1). This increase in aquaculture and the associated demand for ground water in the area, prompted the need for a better understanding of the ground-water resources in the Black Belt area. To address this need, the U.S. Department of Agriculture (USDA), Soil Conservation Service (SCS), is developing a comprehensive program to study the effects of multiple uses of land and water resources in the area. As part of this comprehensive program, the U.S. Geological Survey (USGS), in cooperation with SCS and the Alabama Department of Economic and Community Affairs (ADECA), studied the ground-water resources and inventoried water use for aquaculture in the area. The objectives of that study were to describe (1) the hydrogeology of the major aquifers, (2) the quality of the ground water, (3) the recharge to and discharge from the major aquifers, and (4) the estimated water use for aquaculture in 1990. This report presents the results of the study, which was conducted during 1990-91.

\section{Description of the Study Area}

The study area is in west-central Alabama and encompasses an area of about 2,630 $\mathrm{mi}^{2}$ (square miles). The area includes parts of eight counties: Dallas, Greene, Hale, Marengo, Perry, Pickens, Sumter, and Wilcox (fig. 1). Much of the study area is underlain by black soil and is locally referred to as the Black Belt of Alabama.

The study area lies within the East Gulf Coastal Plain physiographic section and includes parts of five physiographic districts: the Fall Line Hills, the Black Prairie, the Chunnenuggee Hills, the Flatwoods, and the Alluvial Plain (fig. 2). Regional topography in the area dips toward the Gulf of Mexico or the Mississippi embayment. The northern part of the study area, generally north of the line from Eutaw through Greensboro to Marion, lies within the Fall Line Hills district. This district is characterized by flat to moderately-rolling sandy uplands dissected by deeply-entrenched southward and southwestward flowing streams. A 20- to 25-mile-wide crescent-shaped area from the Mississippi State line in southern Pickens and northern Sumter Counties southeastward to Perry County and western Dallas County, is in the Black Prairie district. This district is underlain by black soil for which the Black Belt area gets its name and is characterized by gently- to moderately-rolling prairie with extensive grasslands and few trees. A 5-mile-wide band that extends eastward from Sumter County across Marengo County in northwestern Wilcox County lies in the Chunnenuggee Hills district (fig. 2). This district is characterized by a series of sand hills and cuestas. Most of the hills and cuestas are about 100 to 200 feet higher than the Black Prairie to the north. A lowland about 5 to 8 miles wide, that extends 


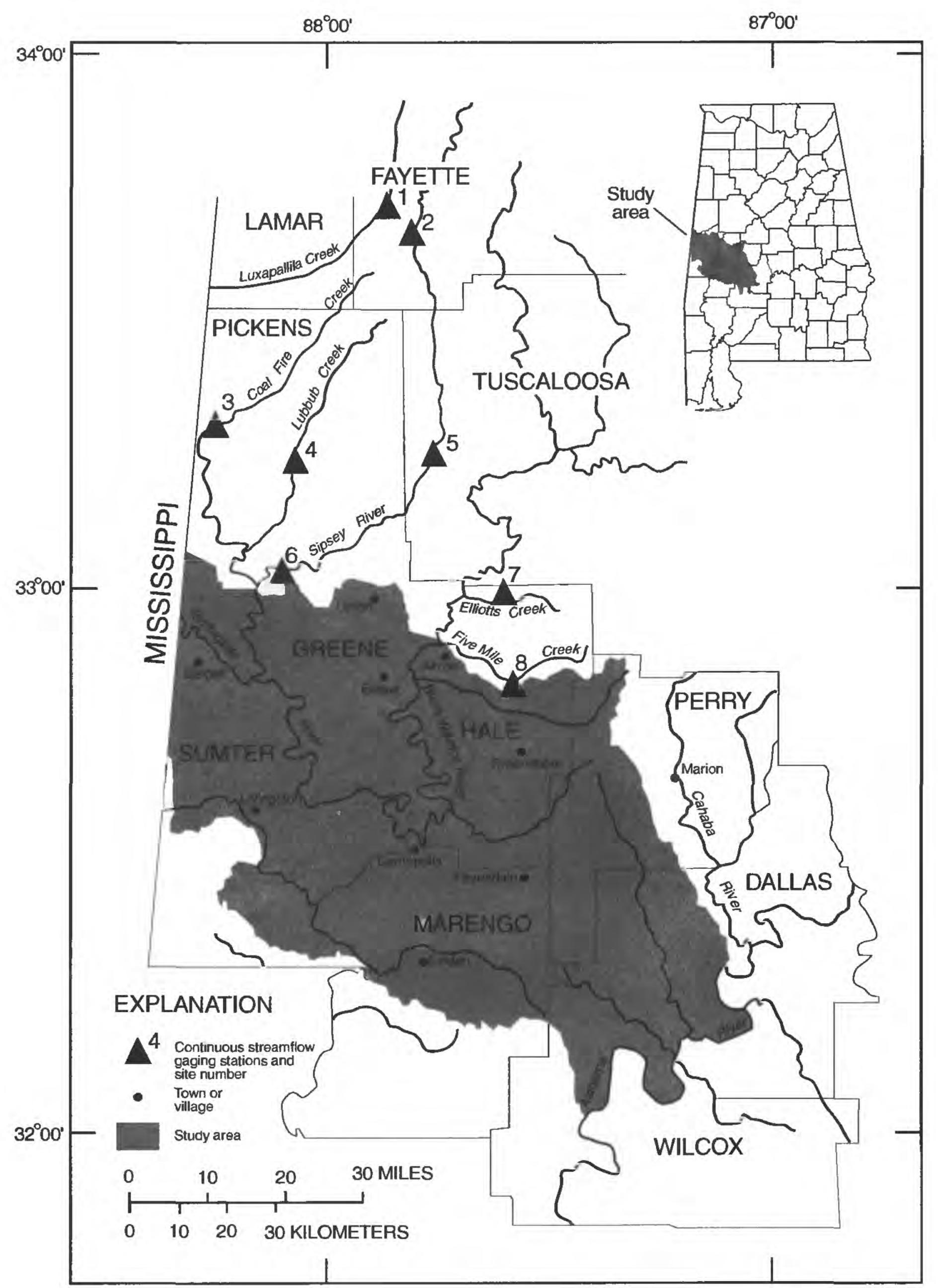

Figure 1. Location of the study area and selected continuous streamflow gaging stations. 


\section{EXPLANATION \\ PHYSIOGRAPHIC DISTRICTS}

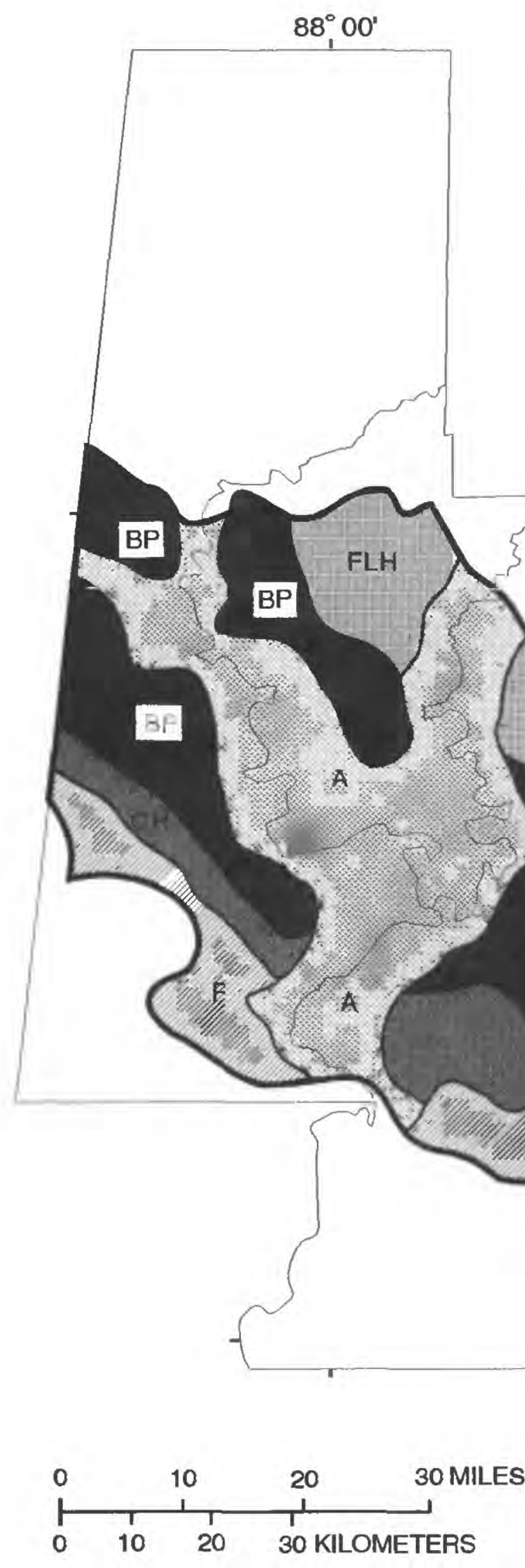

\section{FLH}

Fall Line Hills

\section{BP}

Black Prairie

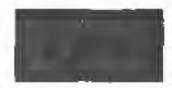

Chunnenuggee Hills

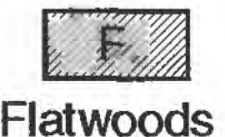

A Alluvial Plain 
from Sumter County through central Marengo County into Wilcox County just east of the Alabama River, lies in the Flatwoods district. The broad, flat, flood plains and terraces that lie along the Tombigbee, Black Warrior, and Alabama Rivers are in the Alluvial Plain. These flood plains are underlain by fluvially deposited clays, silts, and sands.

Because of its proximity to the Gulf of Mexico, west-central Alabama has a moist temperate climate. Annual precipitation amounts are fairly uniform throughout the area. Mean annual rainfall, based on data for period 1970-90, ranges from 6.9 inches for March to 3.4 inches for October (U.S. Department of Commerce, 1970-90).

\section{Methods of Study}

Data collection in this study included inventorying wells, collecting ground-water samples, estimating recharge to and discharge from the major aquifers, and estimating water use for aquaculture. The estimation of water use for aquaculture involved an inventory of commercial catfish ponds. Available data for wells in the study area were compiled and analyzed, and an inventory of large-capacity wells, primarily public-supply wells, was made. The information collected in this well inventory included water-use data, well depths, well yields, aquifers in which the wells were screened and, where possible, water levels. At five of the wells inventoried, water samples were collected to determine the occurrence of common dissolved constituents.

Water samples also were collected from 100 wells throughout the study area to determine the distribution of specific conductance and dissolved chloride concentrations in ground water in the area. Recharge to and discharge from the major aquifers in the study area were estimated from baseflow analysis. Streamflow data from eight daily record sites in the outcrop areas of the major aquifers were analyzed in the baseflow analysis using methods developed by Rutledge (1991, 1992). Water-use requirements for aquaculture were calculated from data obtained from an inventory of catfish ponds. For each of the ponds inventoried, the size, location, topographic setting, surrounding land use, and source of water supply was documented, and an estimate of water use was made.

\section{Previous Investigations}

Reports describing various aspects of the geology, hydrology, and water chemistry in all or part of the study area have been published by the U.S. Geological Survey, the Geological Survey of Alabama, and other agencies. The geology and ground-water resources of Wilcox County have been described by LaMoreaux and Toulmin (1959). Newton and others (1961) reported on the geology and ground-water resources of Marengo County. Wahl (1966) described the geology and ground-water resources of Greene County. The mineral, water, and energy resources of Wilcox 
County have been described by LaMoreaux and others (1969). Newton and others (1971) described the availability of water in Marengo County. Reed (1972) reported on the geology of Perry County, and Reed, Willmon, and Jefferson (1972) described water availability in Perry County. Davis and others (1975) reported on water availability and geology of Hale County. The water availability and geology of Sumter County have been described by Davis, Sanford, and Jefferson (1980). Scott, Golden, and Newton (1981) reported on the geology and water availability of Dallas County. The deposits of Cretaceous and Tertiary age in the study area have been described in reports by Monroe (1941), Copeland (1968), and Davis (1987). Miller (1992) described the general hydrology of the Coastal Plain aquifers.

Other reports describing the geology, hydrology, and water quality in all or part of the study area include those by Mooty (1987), DeJarnette and Crownover (1987), Davis (1987), Barksdale and others (1986a, 1986b), Planert and Sparks (1987), Gardner (1981), and Planert and others (1991). Mooty (1987), and DeJarnette and Crownover (1987) included the study area in their descriptions of the geohydrology and susceptibility of major aquifers to surface contamination. They also presented an inventory of public supply wells and presented potentiometric surface maps of the major aquifers in the area. Davis (1987) and Barksdale and others (1986a, 1986b) presented maps of the potentiometric surfaces and estimated ground-water withdrawals from the Eutaw and Tuscaloosa aquifers (Gordo and Coker aquifers in this report). Planert and Sparkes (1985) estimated the hydraulic conductivity of the confining bed between the Eutaw and Gordo aquifers in Marengo County. Gardner (1981) described the hydraulic characteristics of the Gordo and Eutaw aquifers in west-central Alabama by using a three-dimensional model. Planert and others (1991) developed a regional ground-water flow model for the Southeastern Coastal Plain aquifer system in Alabama, which included aquifers in the study area.

\section{Acknowledgments}

The authors extend their appreciation to the many people who provided information and assistance with this investigation, particularly Perry Oakes, Gregory Whitis, and William Hemstreet of the Alabama Fish Farming Center. Special thanks also go to George Smelley and Barry Bates for allowing access to their ponds for the installation of water-quality monitors. During the well and pond inventory, assistance was provided by the following Soil Conservation Service personnel: Winford Andrews, Gregory Gresham, Andrew Williams, Charles McAlpine, Floyd Wood, Roland Perry, Hugo Bethane, and Anthony Eaton. The Black Belt Water Resources Study Steering Committee provided leadership and guidance throughout the study. Successful completion of the study would not have been possible without the assistance of the catfish farmers and well owners who provided access to their property and furnished well and water-use information. 


\section{HYDROGEOLOGY}

Geologic units that crop out in the study area are shown in figure 3. The units are of sedimentary origin, and consist mainly of sand, clay, gravel, silt, chalk, and sandstone. These deposits range in age from Late Cretaceous to Quaternary. The outcropping units include, in ascending order: the Gordo Formation; the Eutaw Formation; the Selma Group, which consists of the Mooreville and Demopolis Chalks, the Ripley Formation, and the Prairie Bluff Chalk, all of Late Cretaceous age; the Clayton and Porters Creek Formations, undifferentiated; the Naheola Formation; and the Nanafalia Formation, all of Tertiary age. Alluvial and terrace deposits of Quaternary age overlie the older deposits in, and adjacent to, the flood plains of the Tombigbee, Black Warrior, and Alabama Rivers, and some of the larger streams in the study area.

The Coker Formation crops out north of the study area and underlies the Gordo Formation (fig. 4). Rocks of Early Cretaceous age do not crop out in Alabama, but thin northward and pinch out in the subsurface near the northern boundary of the study area (fig. 5). Pink nodular limestone fragments and red and green shale, found near the top of the Lower Cretaceous rocks, distinguish them from the massive sand of the overlying Coker Formation of Late Cretaceous age. The Cretaceous deposits overlie Paleozoic sedimentary rocks.

The water-bearing characteristics of the geologic units that underlie the study area are described in figure 6. The water-bearing characteristics described for each geologic unit apply to one or more sand or sand-and-gravel beds within the unit. The beds of permeable materials within an aquifer commonly are hydraulically connected, but in some instances, are separated by layers of low-permeability clay, chalk, limestone, or other material. No attempt is made in this report to delineate the occurrence of these sand and gravel beds within each formation.

\section{Deposits of Quaternary Age}

Deposits of Quaternary age overlie older formations throughout a large part of the study area (fig. 3). These deposits consist of alluvial and terrace deposits associated with the flood plains of present and ancestral large streams. They consist mainly of gravel, sand, silt, and clay. The alluvial deposits generally range in thickness from 0 to $60 \mathrm{feet}$, but are as much as 80 feet thick in some places. Terrace deposits are as much as 100 feet thick.

Data for 12 wells tapping the deposits of Quarternary age are given in table 1 (plate 2). Terrace deposits yield sufficient quantities of water for domestic supplies. Alluvial sediments are a potential source of large supplies where sand beds are of sufficient saturated thickness. 
EXPLANATION

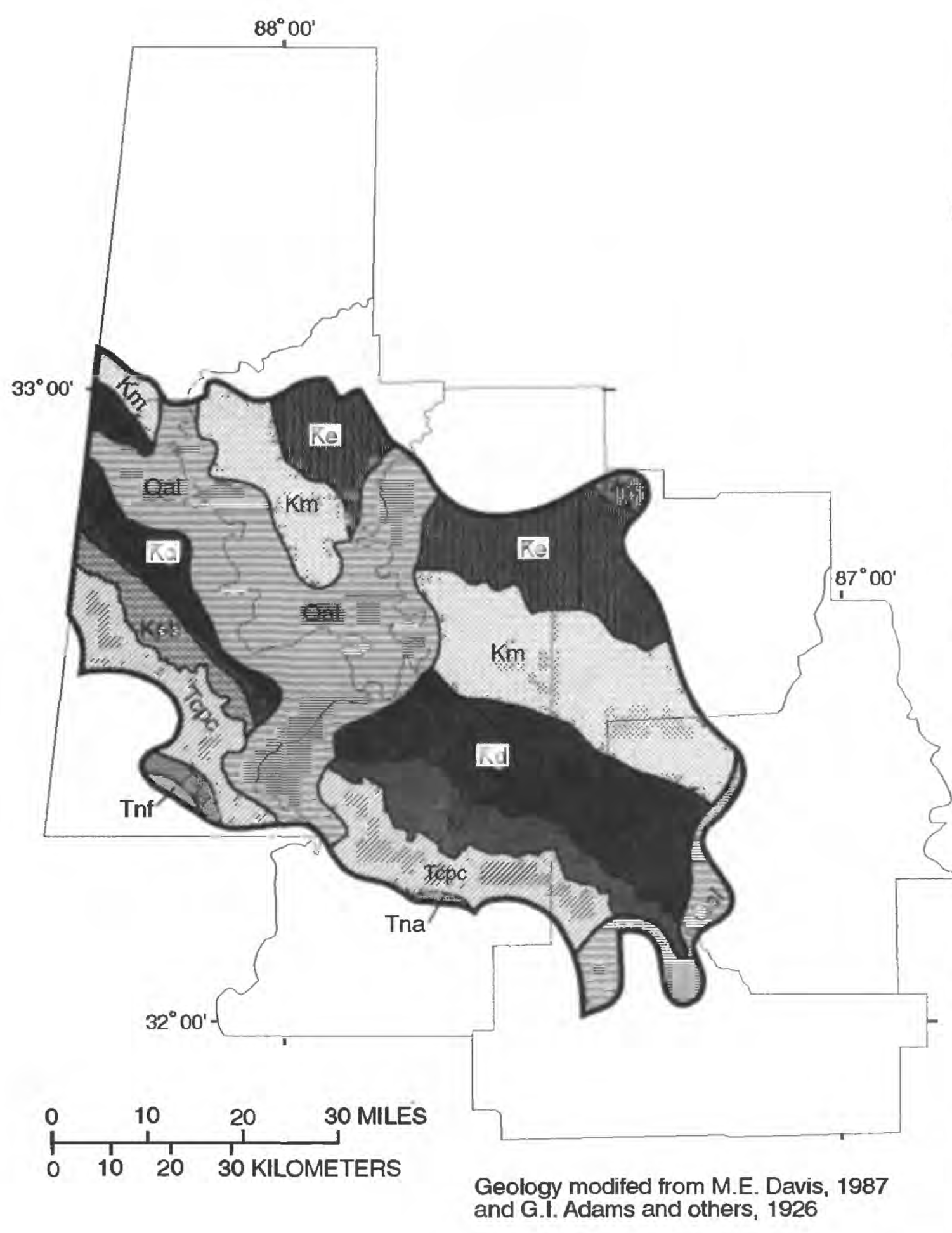

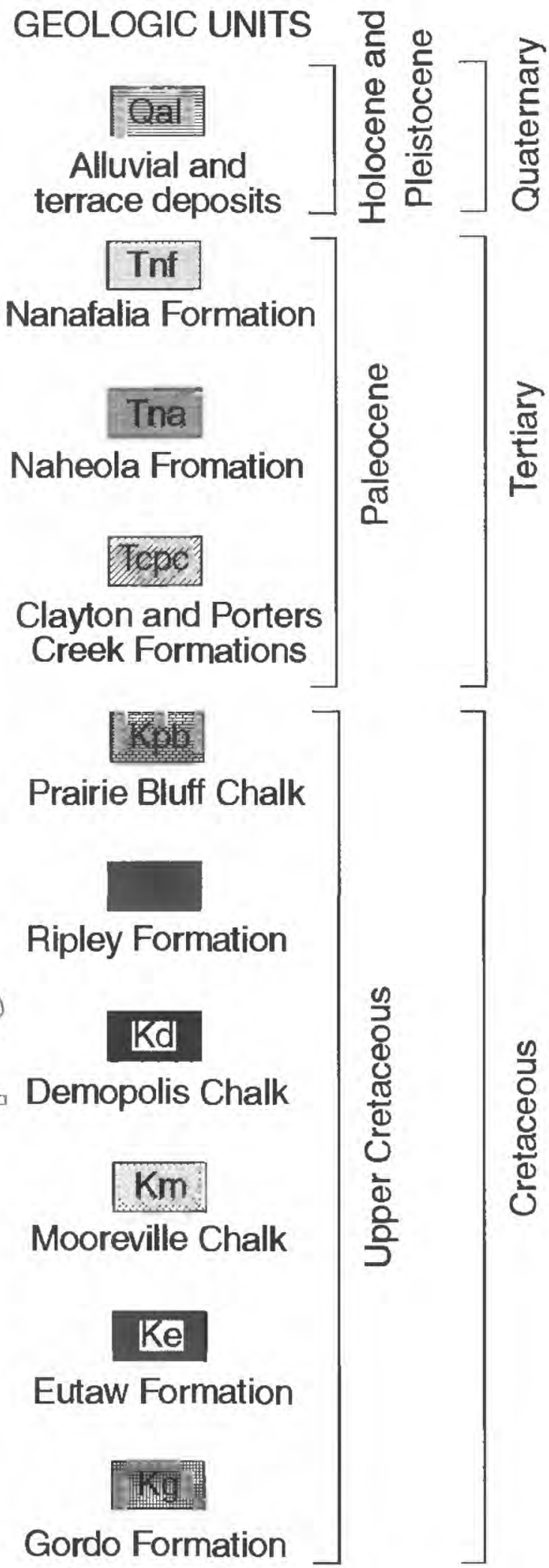

Figure 3. Generalized geology of the Black Belt area of west-central Alabama. 


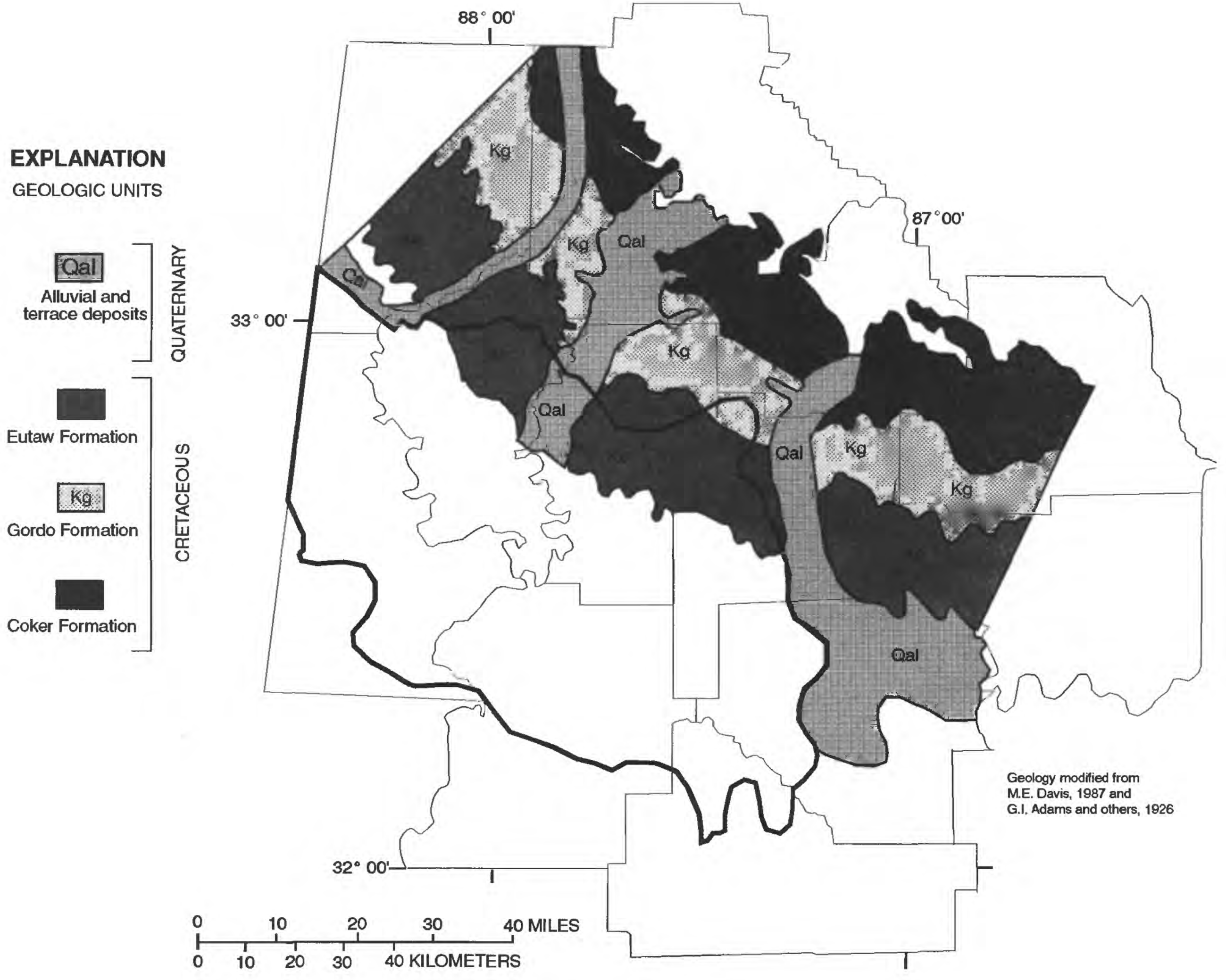

Figure 4. Estimated areas of recharge of the major aquifer in the Black Belt area of west-central Alabama. 


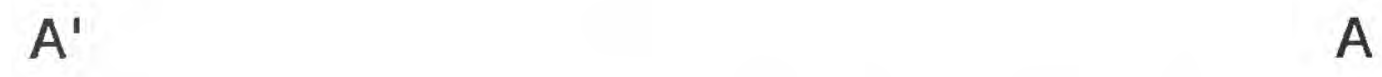

EXPLANATION

GEOLOGIC UNITS

Clayton and Porters
Creek Formations

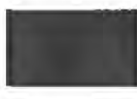

Ripley Formation

Kd Demopolis Chalk

Km: Mooreville Chalk

Eutaw Formation

Ka Gordo Formation

Kc Coker Formation

Lower Cretaceous
rocks

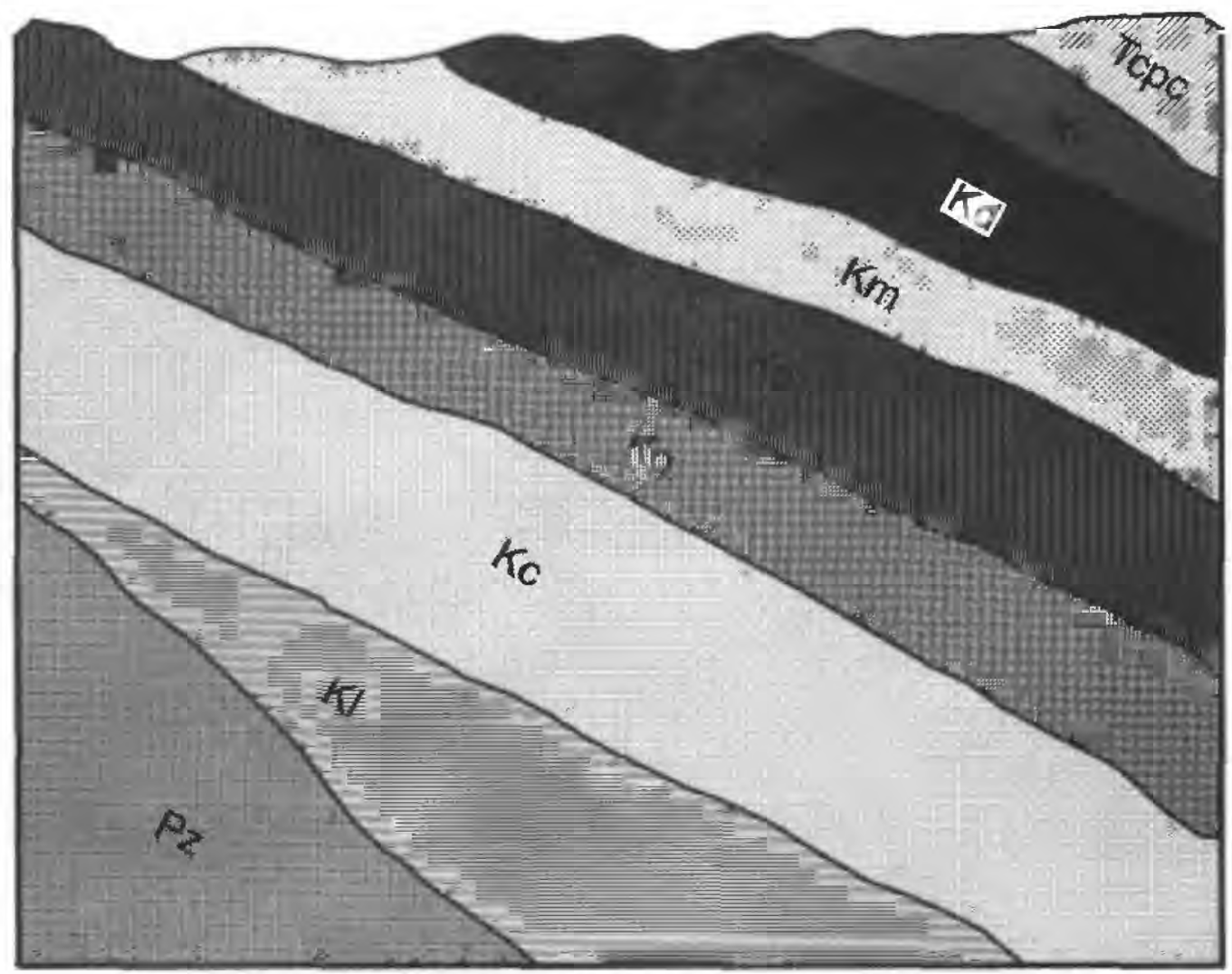

Modified from M.E. Davis, 1980 Vertical scale greatly exaggerated

PZ Paleozoic rocks
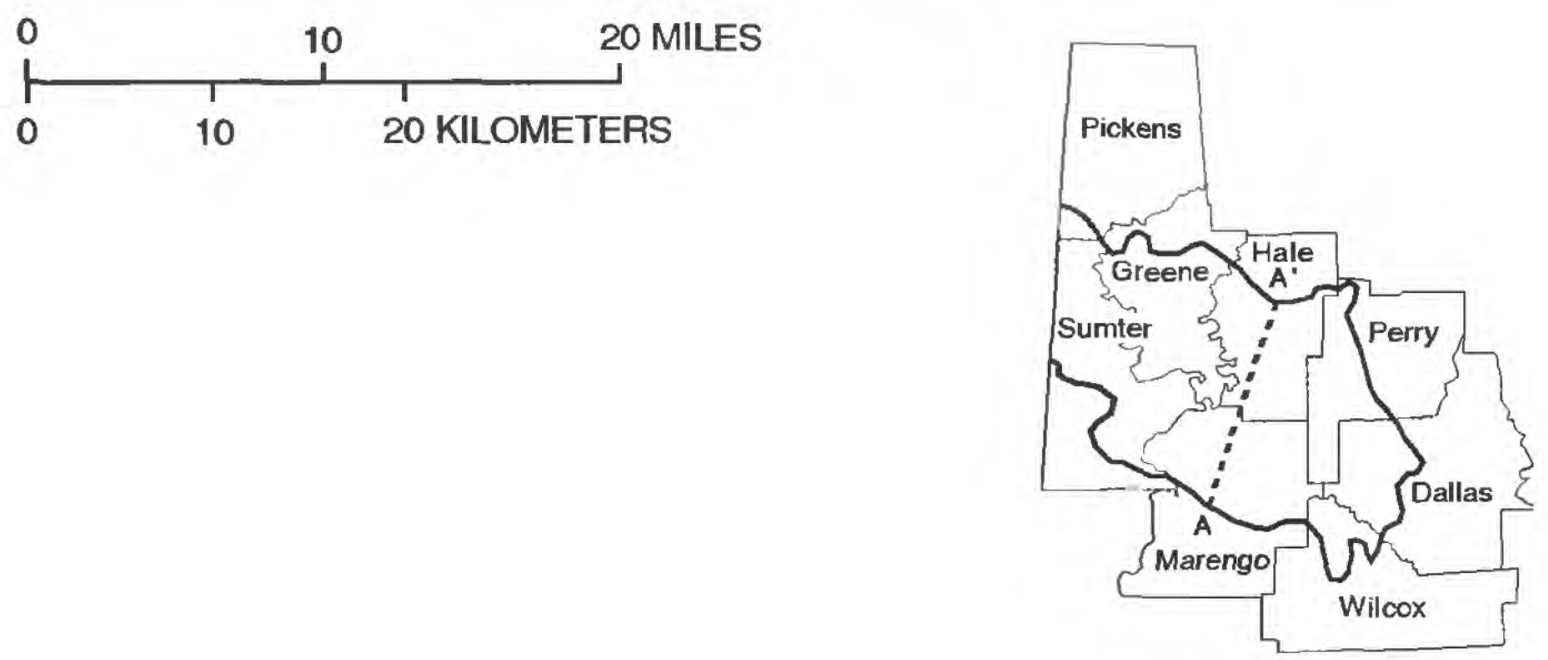

Figure 5. Diagrammatic geologic section of the Black Belt area of west-central Alabama. 


\begin{tabular}{|c|c|c|c|c|c|c|c|c|}
\hline $\begin{array}{l}\text { Era- } \\
\text { them }\end{array}$ & System & Series & Group & Geologic unit & $\begin{array}{l}\text { Thickness } \\
\text { (feet) }\end{array}$ & Lithoiogy & Aquifer & Water-bearing properties \\
\hline \multirow{6}{*}{ 总 } & \multirow[t]{2}{*}{ 窎 } & 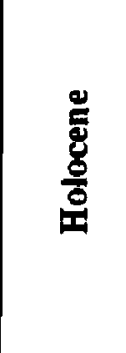 & \multirow{3}{*}{ है } & Alluvium & $0-80$ & $\begin{array}{l}\text { Clay, silt, sand, } \\
\text { and gravei }\end{array}$ & $\begin{array}{l}\text { Alluvial } \\
\text { aquifer }\end{array}$ & $\begin{array}{l}\text { Supplies water to shailow dug } \\
\text { and driven welis iu the flood } \\
\text { piains of the major streams aud } \\
\text { rivers. Adjacent to major } \\
\text { streams, where luduced recharge } \\
\text { is possihle, large quantities of } \\
\text { water can be withdrawn from } \\
\text { these beds. }\end{array}$ \\
\hline & & 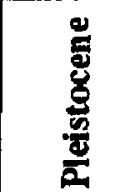 & & $\begin{array}{l}\text { Terrace } \\
\text { deposits }\end{array}$ & $0-100$ & $\begin{array}{l}\text { Clay, silt, sand, } \\
\text { and gravel }\end{array}$ & $\begin{array}{l}\text { Shallow } \\
\text { aquifer }\end{array}$ & $\begin{array}{l}\text { Will yield } 10 \mathrm{gal} / \mathrm{miu} \text { or more } \\
\text { to indl vidual weils where } \\
\text { saturated sauds are of sufficient } \\
\text { thickness. }\end{array}$ \\
\hline & \multirow{4}{*}{ 赵 } & \multirow{4}{*}{ 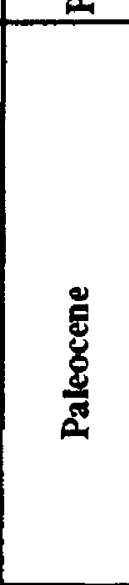 } & & \begin{tabular}{|l|} 
Nanafalia \\
Formation
\end{tabular} & $\overline{150-200}$ & $\begin{array}{l}\text { Saud, saudy mari, } \\
\text { sandy clay, and } \\
\text { Ifgnite }\end{array}$ & $\begin{array}{l}\text { Nauafaila } \\
\text { aquifer }\end{array}$ & $\begin{array}{l}\text { Source of puhlic supplies } \\
\text { south of the study area. }\end{array}$ \\
\hline & & & \multirow{3}{*}{ 窇 } & $\begin{array}{l}\text { Naheoia } \\
\text { Formation }\end{array}$ & 100-190 & $\begin{array}{l}\text { Siity clay, fine- } \\
\text { grained saud, } \\
\text { lignite, laminated } \\
\text { sand aud ciay }\end{array}$ & $\begin{array}{l}\text { Naheola } \\
\text { aquifer }\end{array}$ & $\begin{array}{l}\text { Welis south of the study area } \\
\text { reportediy produce as much } \\
\text { as } 50 \mathrm{gal} / \mathrm{min} \text {. }\end{array}$ \\
\hline & & & & \multirow{2}{*}{\begin{tabular}{l|} 
Porters \\
Creek \\
aud \\
Clayton \\
Formations
\end{tabular}} & \multirow[t]{2}{*}{$270-520$} & \multirow{2}{*}{$\begin{array}{l}\text { Massive } \\
\text { marine ciay } \\
\text { Chalky marl, } \\
\text { limestone and } \\
\text { conglomerate, } \\
\text { thin basal sand }\end{array}$} & & Not a source of water. \\
\hline & & & & & & & $\begin{array}{l}\text { Clayton } \\
\text { aquifer }\end{array}$ & Yields less thau 10 gai $/ \mathrm{min}$. \\
\hline \multirow{8}{*}{$\frac{.}{8}$} & \multirow{8}{*}{ 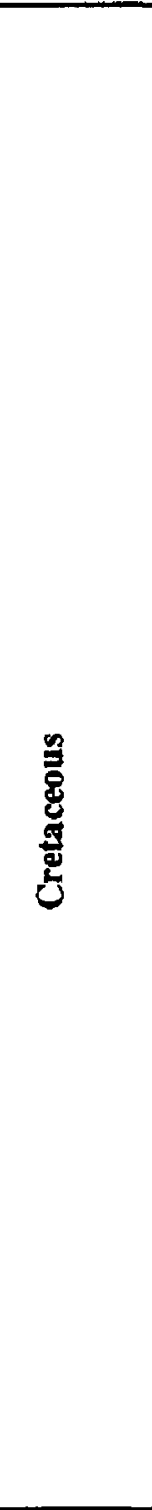 } & \multirow{7}{*}{ 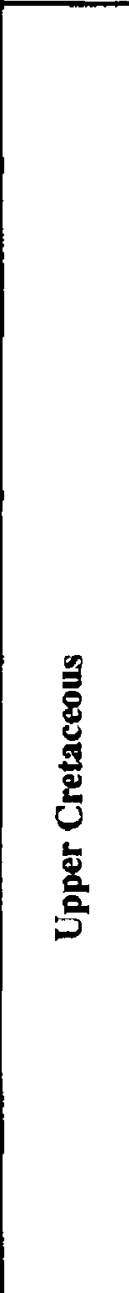 } & \multirow{4}{*}{ 胥 } & $\begin{array}{l}\text { Prairie } \\
\text { Bluff } \\
\text { Chalk } \\
\end{array}$ & 50-100 & $\begin{array}{l}\text { Massive, compact } \\
\text { chalk and caicareous } \\
\text { sandy ciay }\end{array}$ & & Not a source of water. \\
\hline & & & & $\begin{array}{l}\text { Ripeiy } \\
\text { Formation }\end{array}$ & 35-300 & $\begin{array}{l}\text { Calcareous sand- } \\
\text { stone, sandy chaik, } \\
\text { sandy caicareons } \\
\text { clay, and fossiliferous } \\
\text { sandstone } \\
\end{array}$ & $\begin{array}{l}\text { Ripiey } \\
\text { aquifer }\end{array}$ & $\begin{array}{l}\text { Minor source of water. } \\
\text { Generally yields } 10 \mathrm{gal} / \mathrm{min} \\
\text { or iess. }\end{array}$ \\
\hline & & & & $\begin{array}{l}\text { Demopolis } \\
\text { Chaik }\end{array}$ & 200-500 & $\begin{array}{l}\text { Fossiliferous chaik; } \\
\text { caicareous clay }\end{array}$ & & Not a source of water. \\
\hline & & & & $\begin{array}{l}\text { Mooreville } \\
\text { Chalk }\end{array}$ & $350-420$ & $\begin{array}{l}\text { Silty chalk, } \\
\text { calcareous clay; } \\
\text { thin iayers of } \\
\text { limestone and } \\
\text { caicareous sandstone } \\
\end{array}$ & & Not a source of water. \\
\hline & & & & $\begin{array}{l}\text { Eutaw } \\
\text { Formation }\end{array}$ & 400 & $\begin{array}{l}\text { Fine- to coarse- } \\
\text { grained sand, } \\
\text { ciay and chaiky } \\
\text { sand } \\
\end{array}$ & $\begin{array}{l}\text { Eutaw } \\
\text { aquifer }\end{array}$ & $\begin{array}{l}\text { Primary source of ground } \\
\text { water in the study area. } \\
\text { Reportediy will yield } 2 \mathrm{Mgal} / \mathrm{d} \\
\text { or more to individuai wells. } \\
\end{array}$ \\
\hline & & & \multirow{2}{*}{ 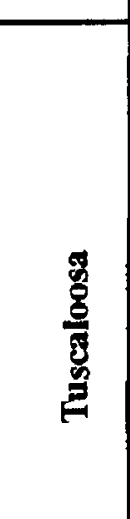 } & $\begin{array}{l}\text { Gordo } \\
\text { Formation }\end{array}$ & $300-450$ & $\begin{array}{l}\text { Reddish-yellow } \\
\text { sand and purple } \\
\text { mottled ctay; } \\
\text { poorly-sorted } \\
\text { coarse-grained } \\
\text { sand and gravel } \\
\text { in lower part } \\
\end{array}$ & $\begin{array}{l}\text { Gordo } \\
\text { aquifer }\end{array}$ & $\begin{array}{l}\text { Excelleut sonrce of water. } \\
\text { Reportedly will yleld } 2 \mathrm{Mgai} / \mathrm{d} \\
\text { or more to ludividuai weils. }\end{array}$ \\
\hline & & & & $\begin{array}{l}\text { Coker } \\
\text { Formation }\end{array}$ & $500 \cdot 1,000$ & $\begin{array}{l}\text { Sand, gravel and } \\
\text { clay. Coarser } \\
\text { sand heds and } \\
\text { gravel heds near } \\
\text { base } \\
\end{array}$ & $\begin{array}{l}\text { Coker } \\
\text { aquifer }\end{array}$ & $\begin{array}{l}\text { Potential source of } 1 \mathrm{Mgal} / \mathrm{d} \\
\text { or more to individual wells. }\end{array}$ \\
\hline & & \multicolumn{3}{|c|}{ Lower Cretaceous rocks } & $0-1,000$ & $\begin{array}{l}\text { Pink nodular } \\
\text { limestone } \\
\text { fragments and } \\
\text { red and green } \\
\text { shale near top } \\
\end{array}$ & & \\
\hline 䒴 & \multicolumn{4}{|c|}{ Pennsylvanian to Cambrian rocks } & $1,000_{+}$ & $\begin{array}{l}\text { Sandstone, shale, } \\
\text { limestone, doiomite, } \\
\text { and chert }\end{array}$ & & \\
\hline
\end{tabular}

Figure 6. Correlation chart showing geologic units and aquifers in the Black Belt area of west-central Alabama, and their water-bearing properties. 


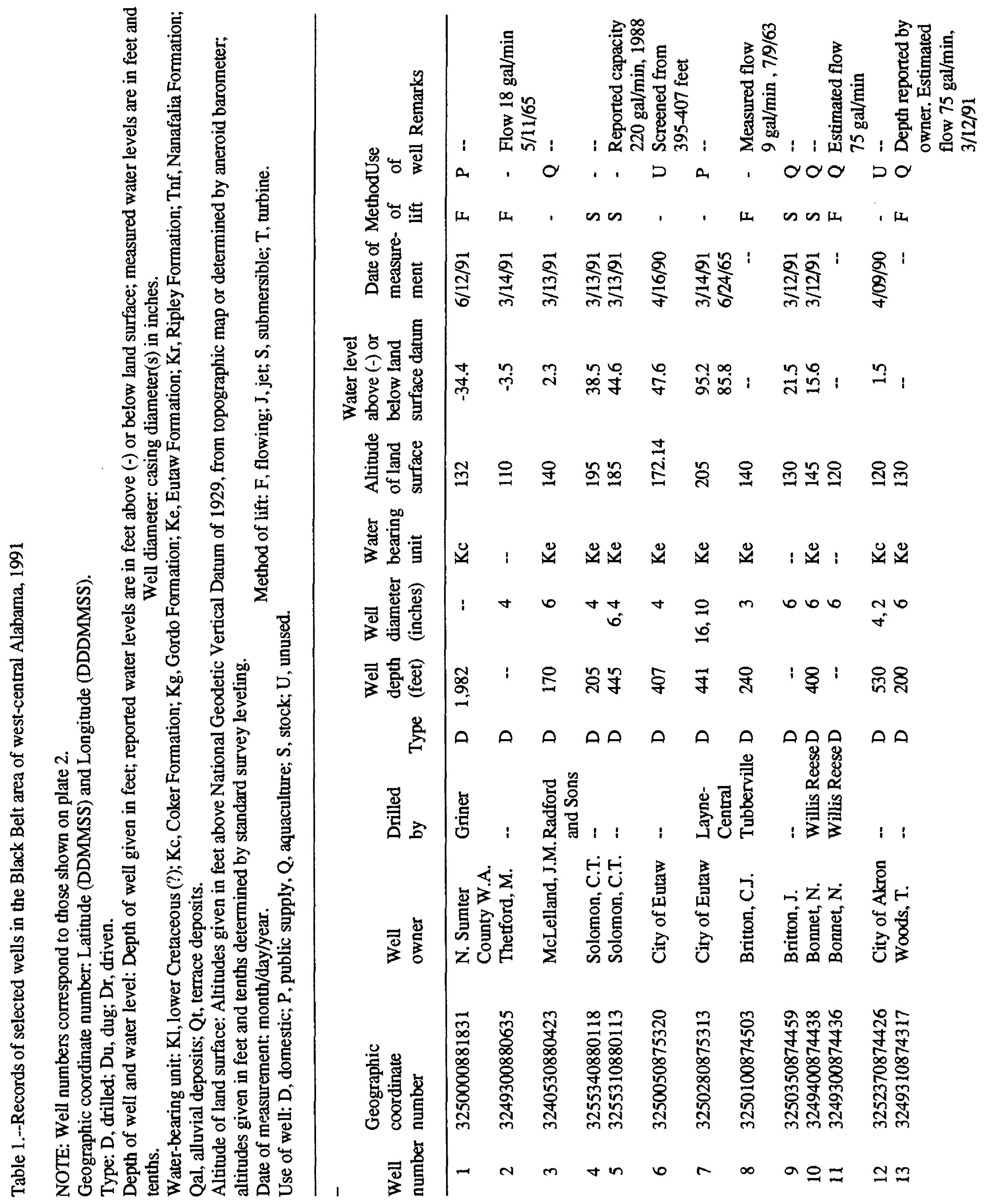




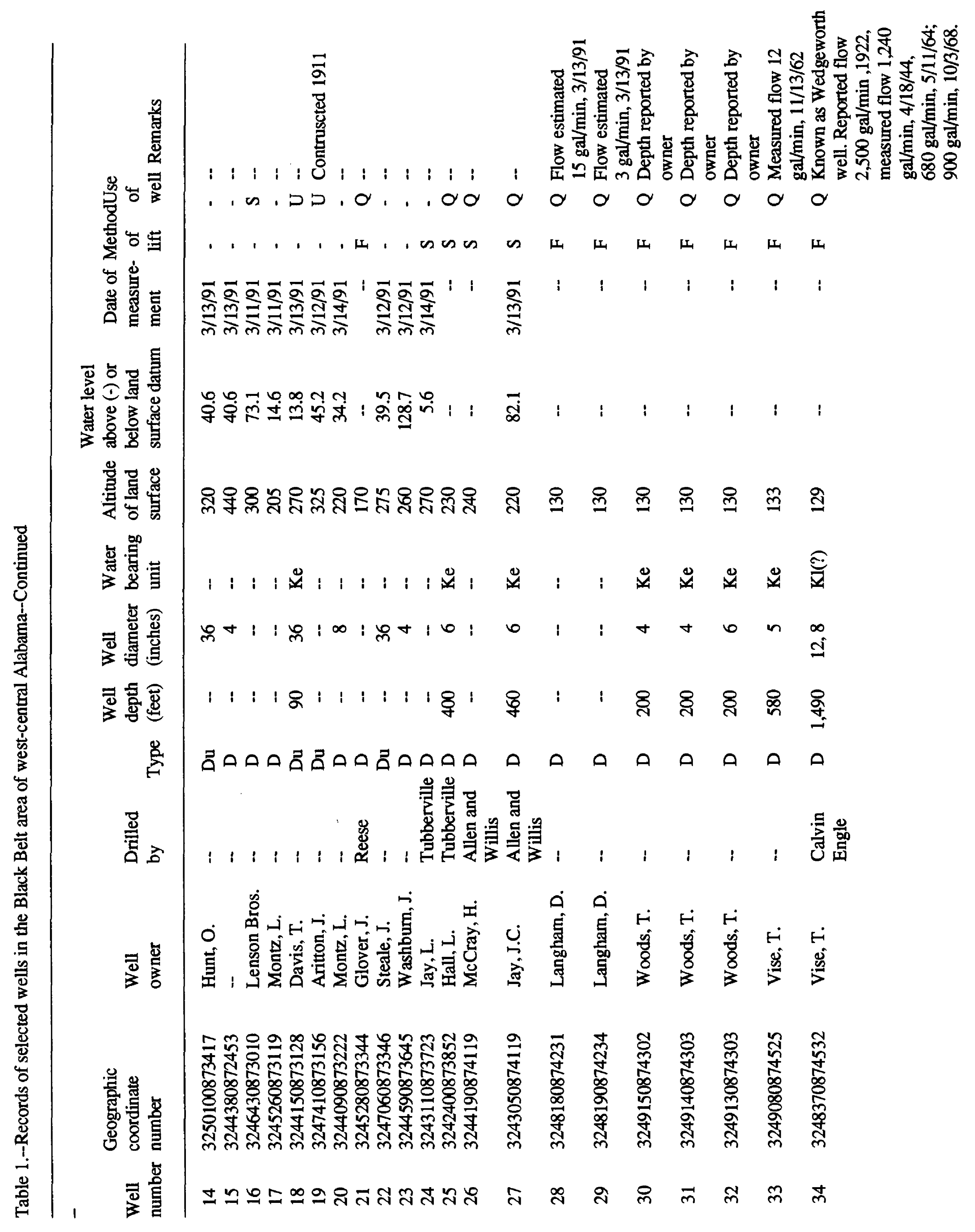




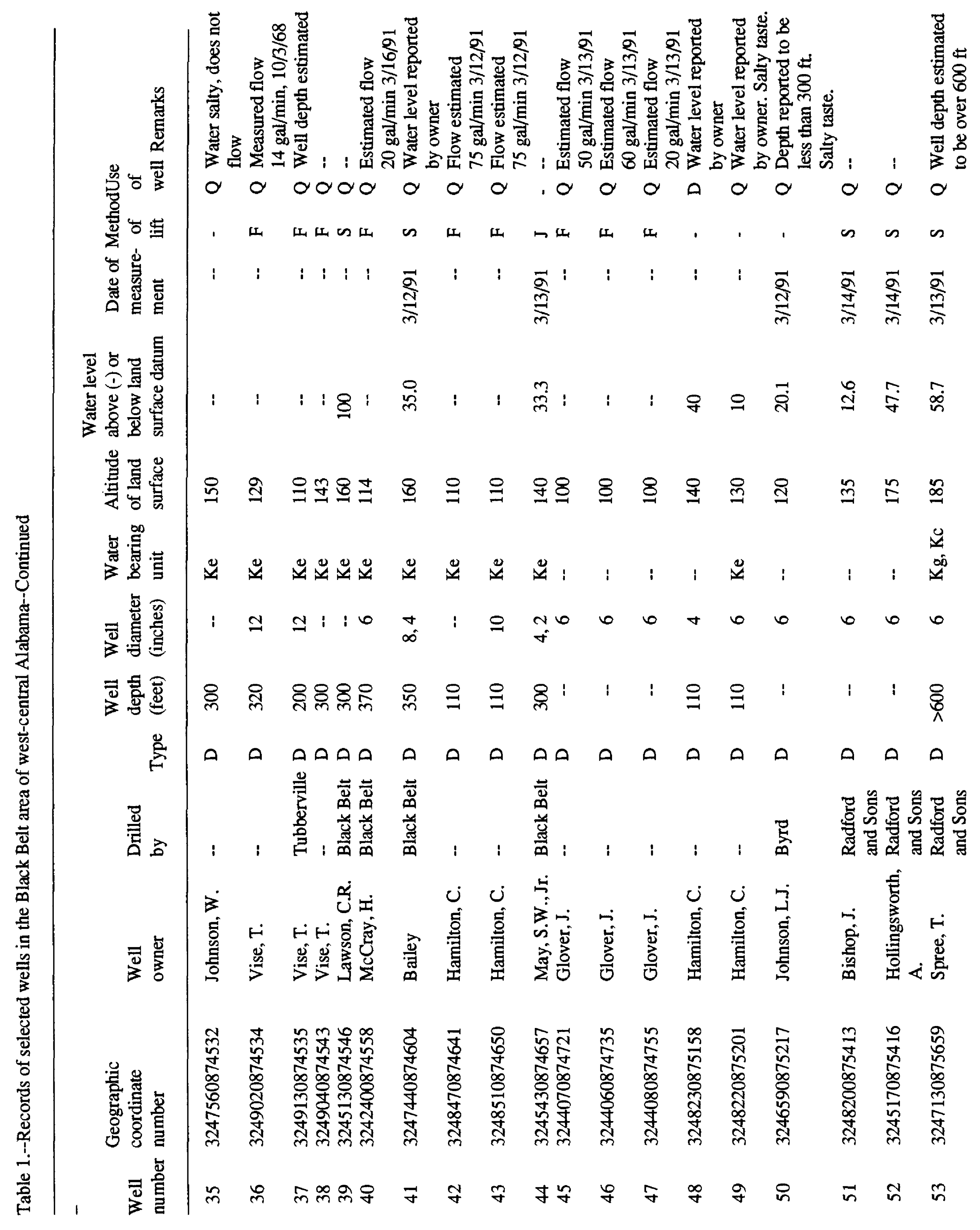




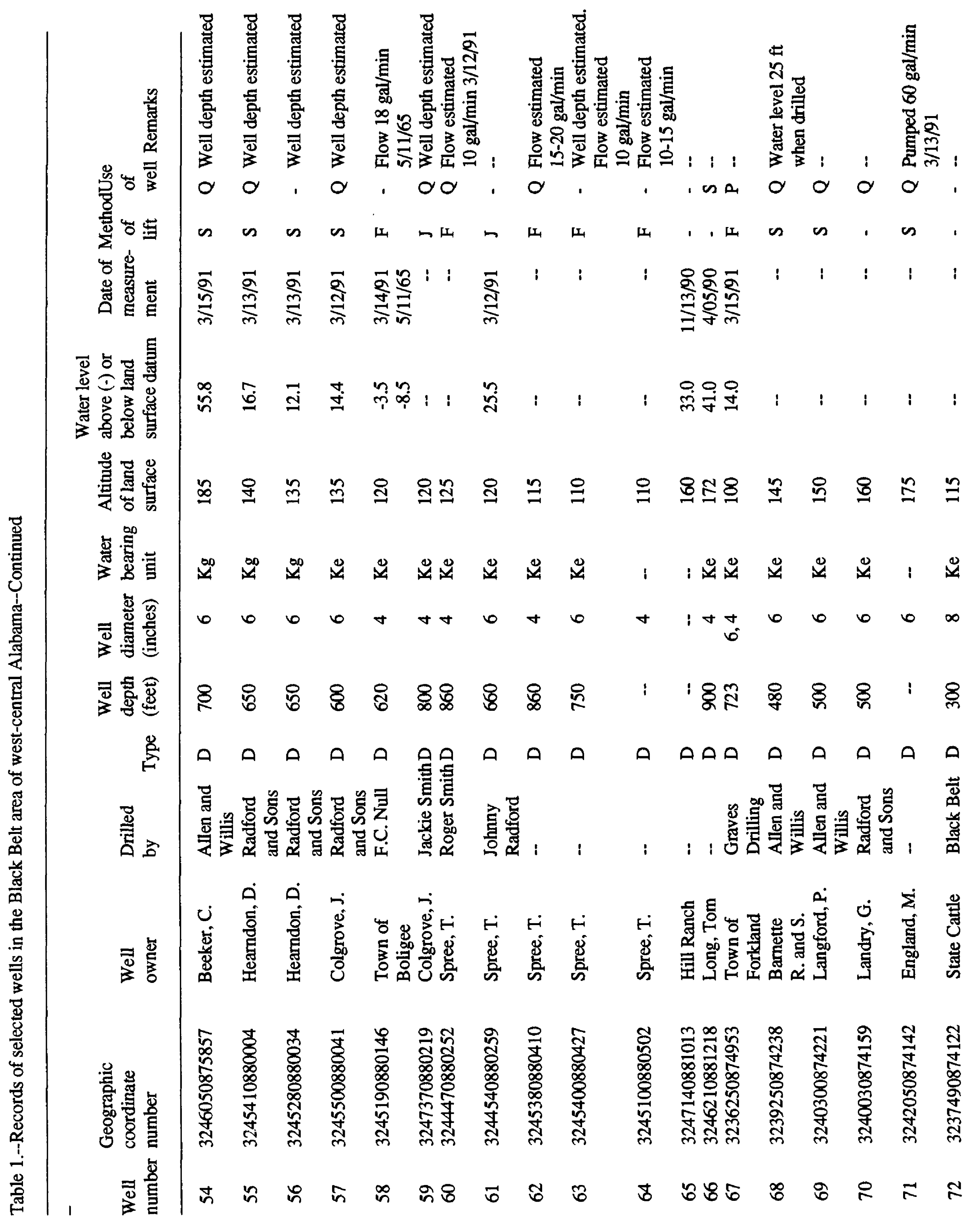


:

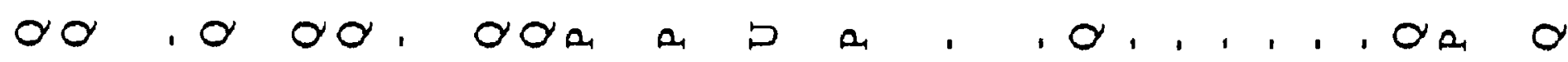

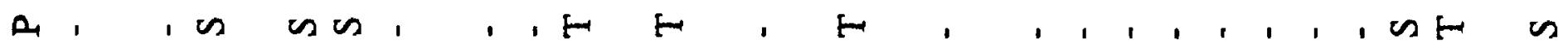

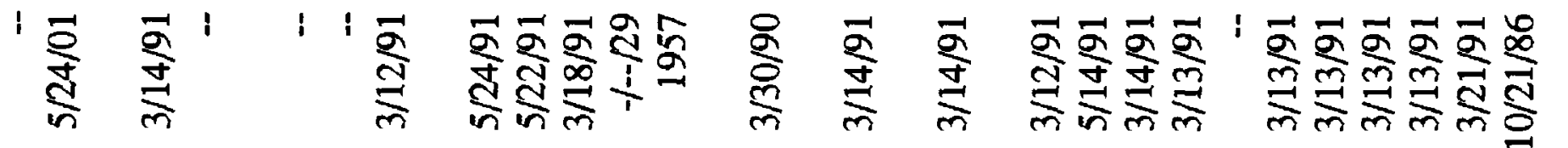

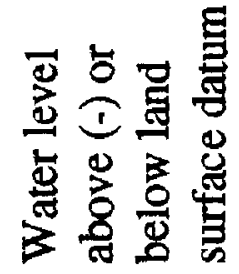

营营尊

总腒

焉离兽

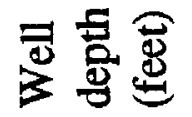

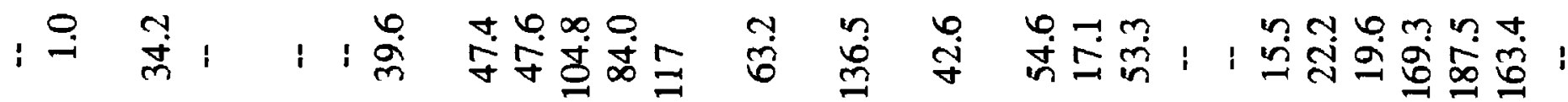

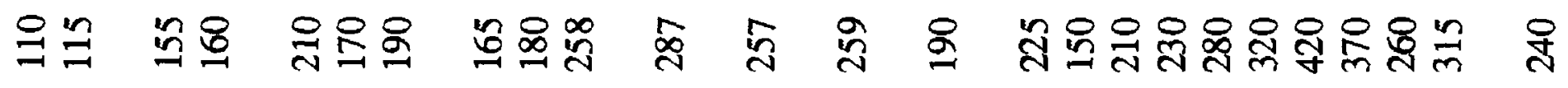

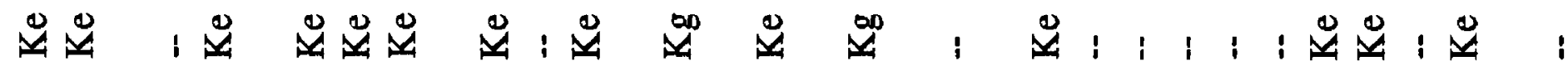

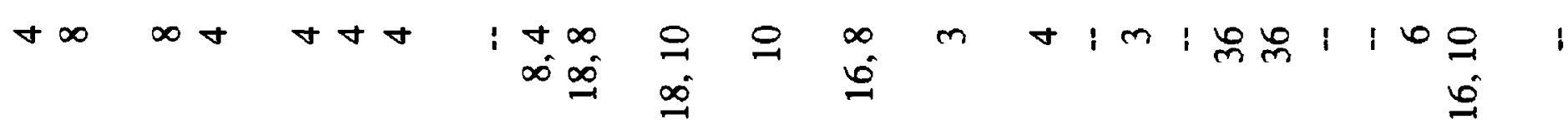

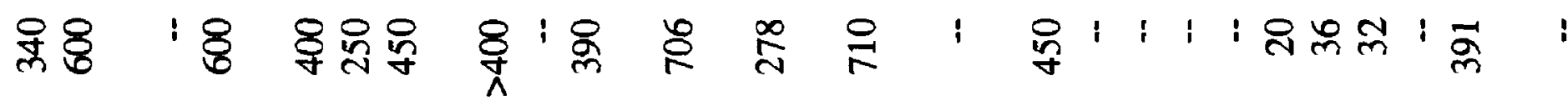

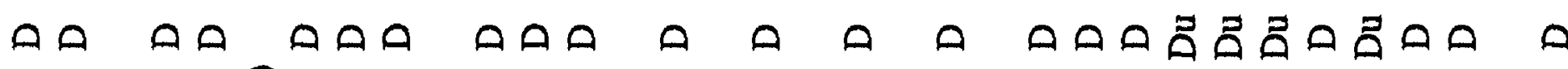

泀

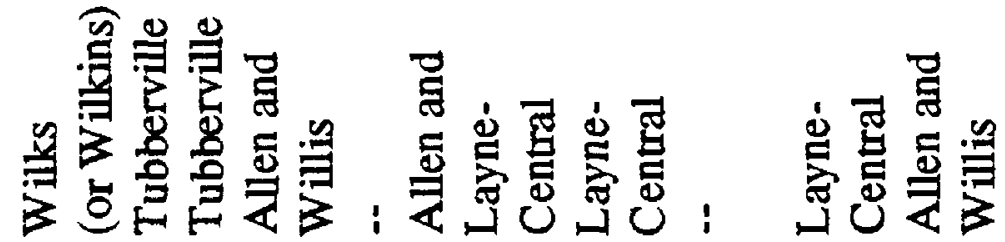
离到

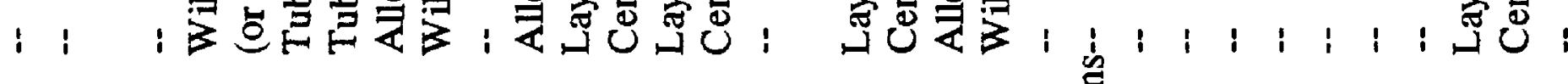

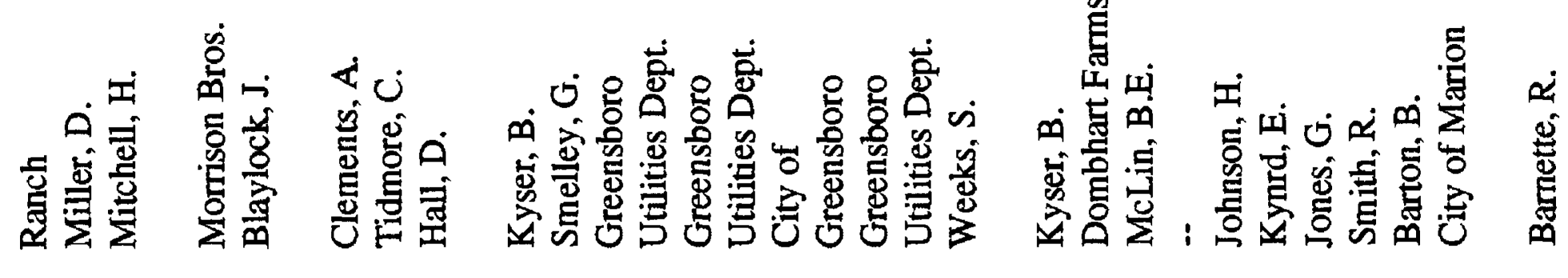

言咅

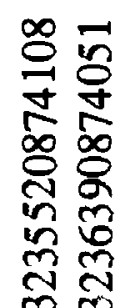

S.

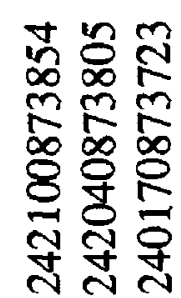

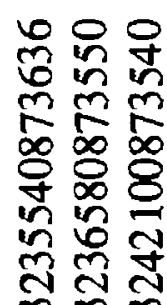

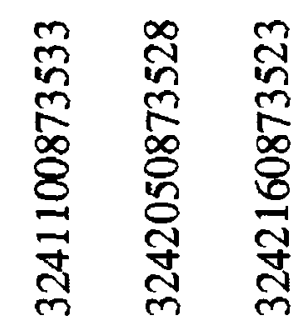

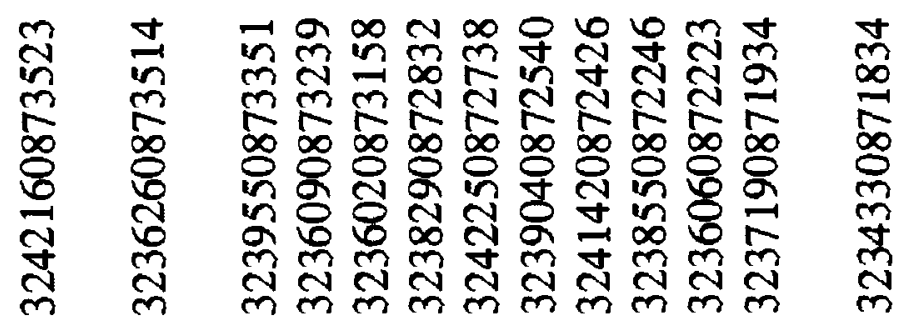

总

$m$

in

N臬 $\infty$ 


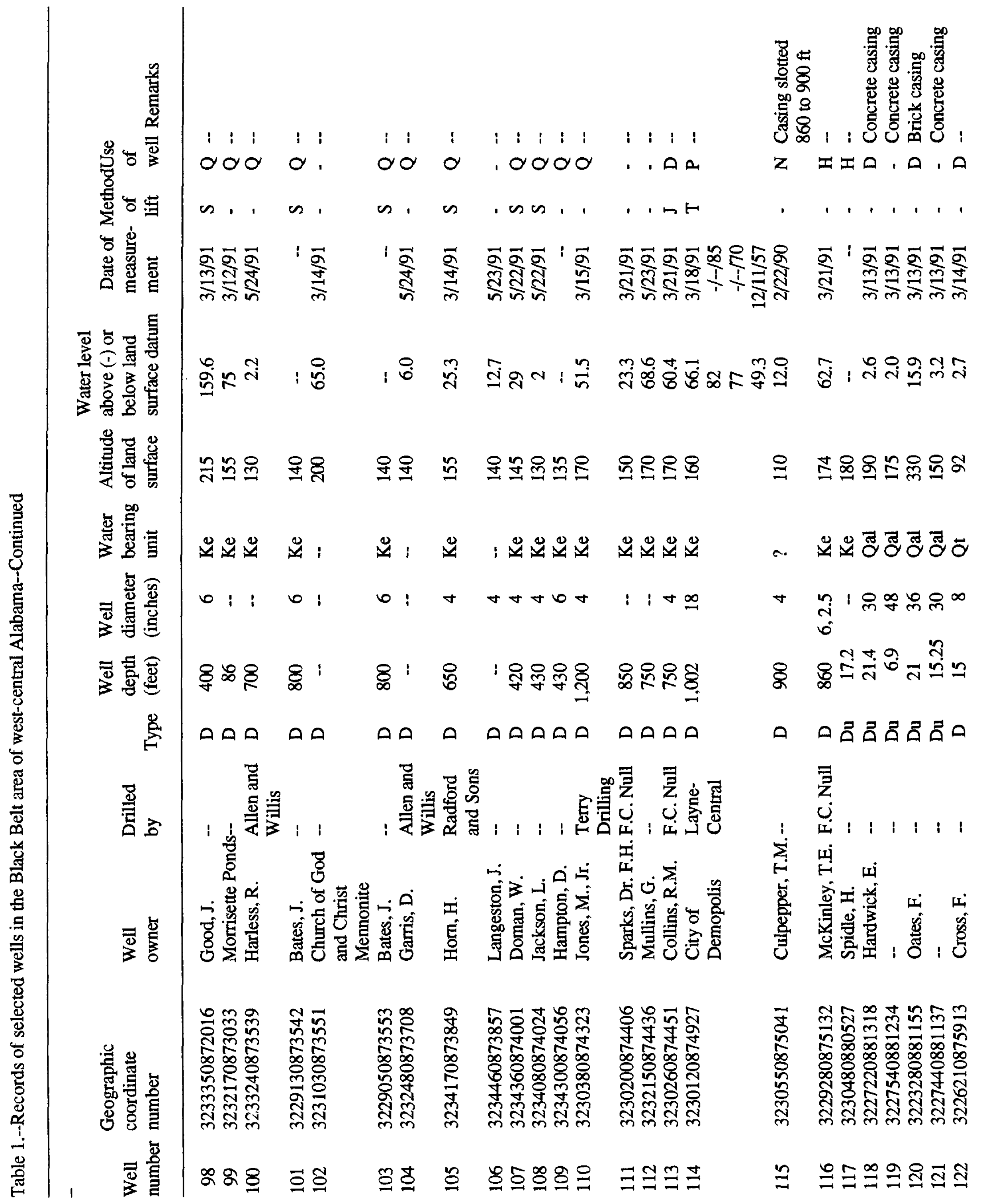


总芦苔

总

畓

a a da an a dodóa

홀

宂

苋农

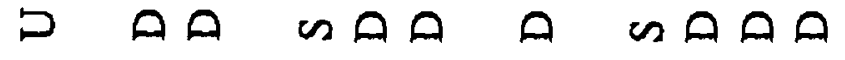

$H \quad, \quad \infty$

,

, cosenes. in

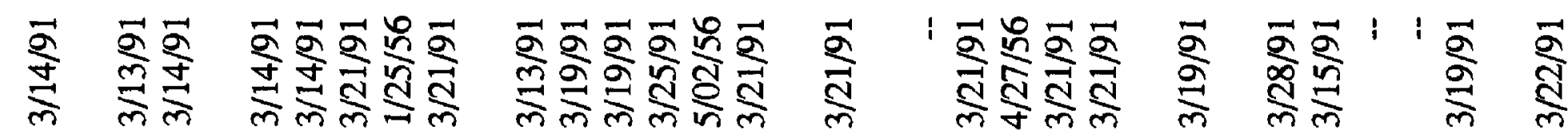

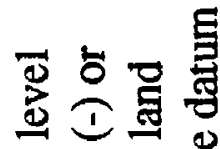

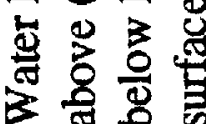

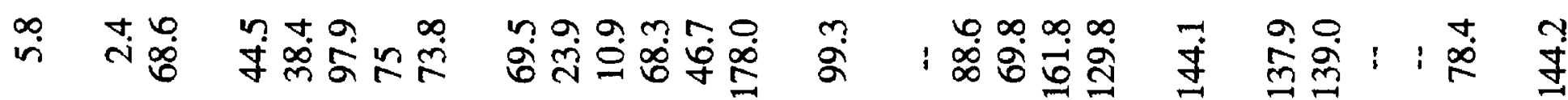

营焉莺

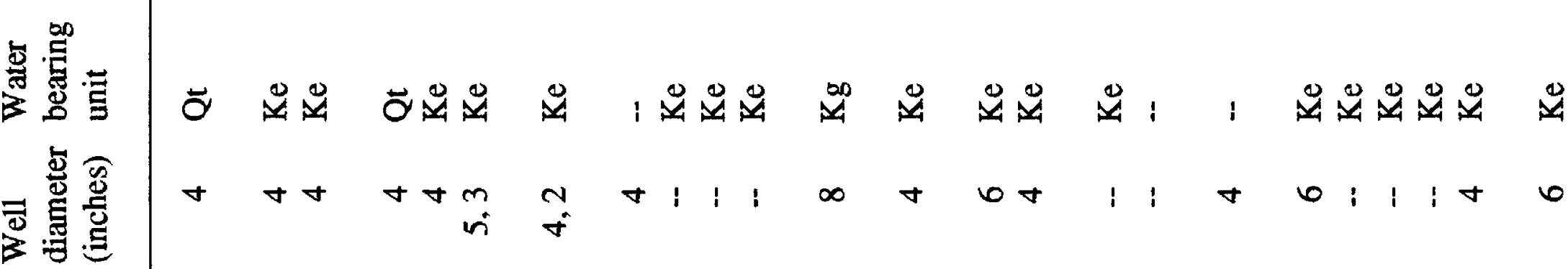

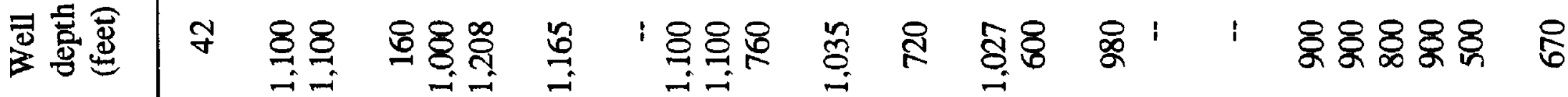
F 兽

实

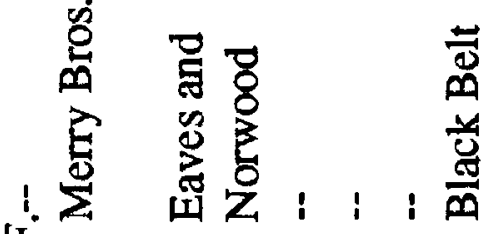

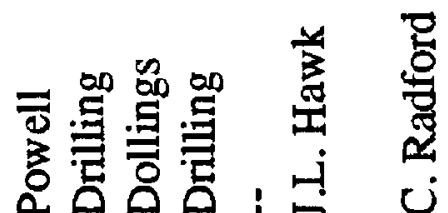

홍

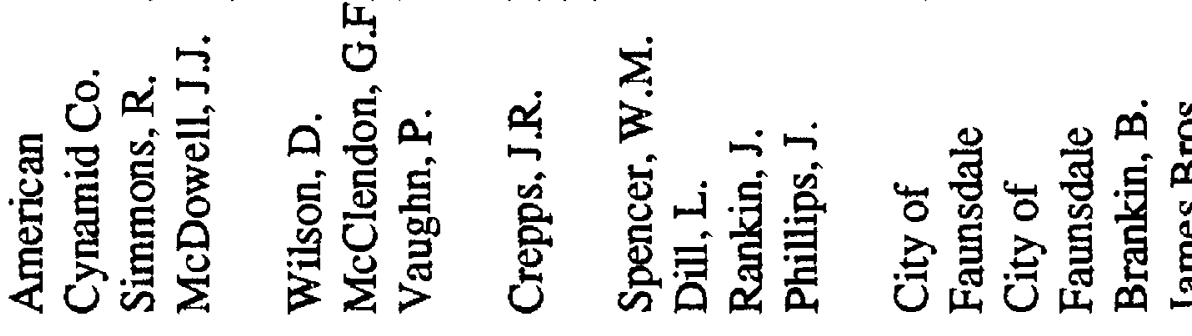

卷

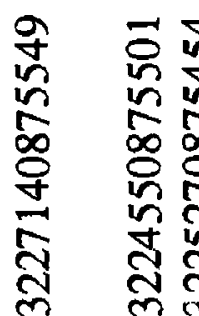

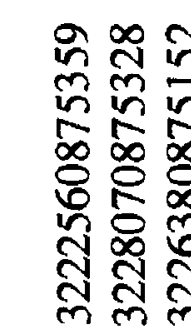

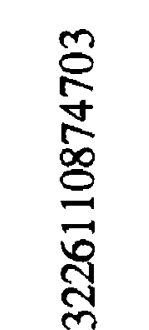

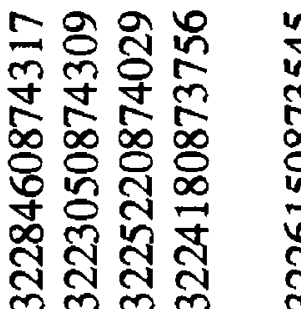

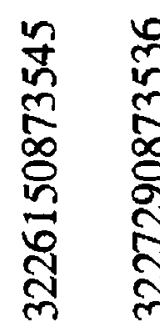

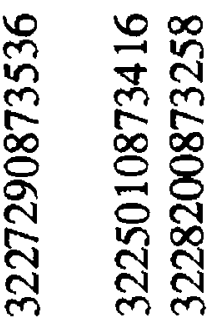

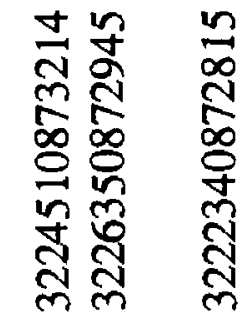

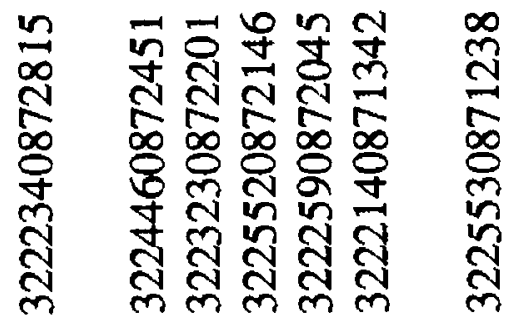

क若

ฮิ

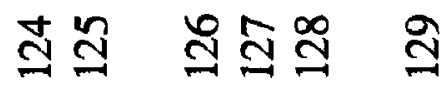

요요

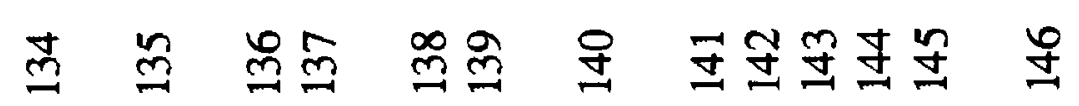




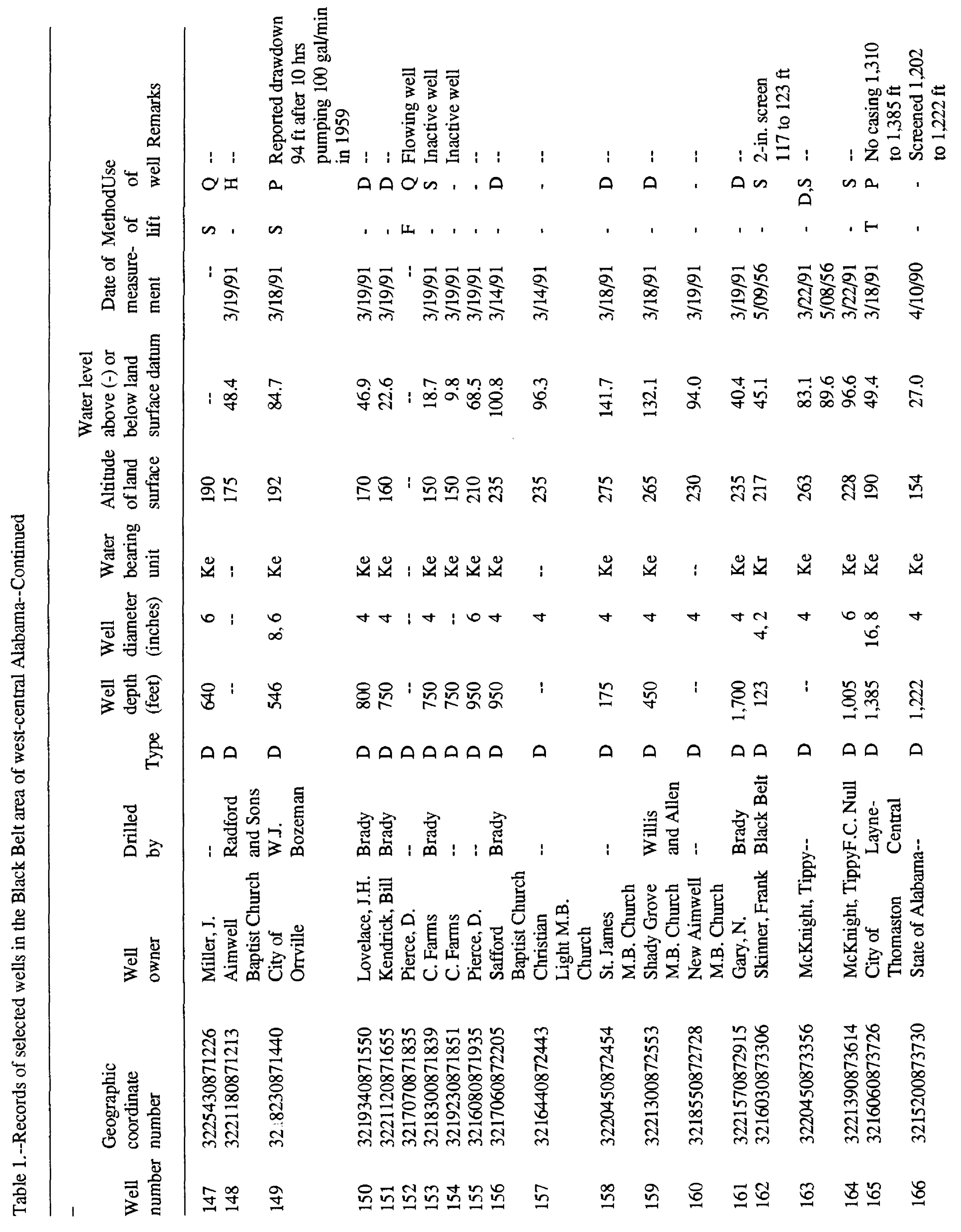




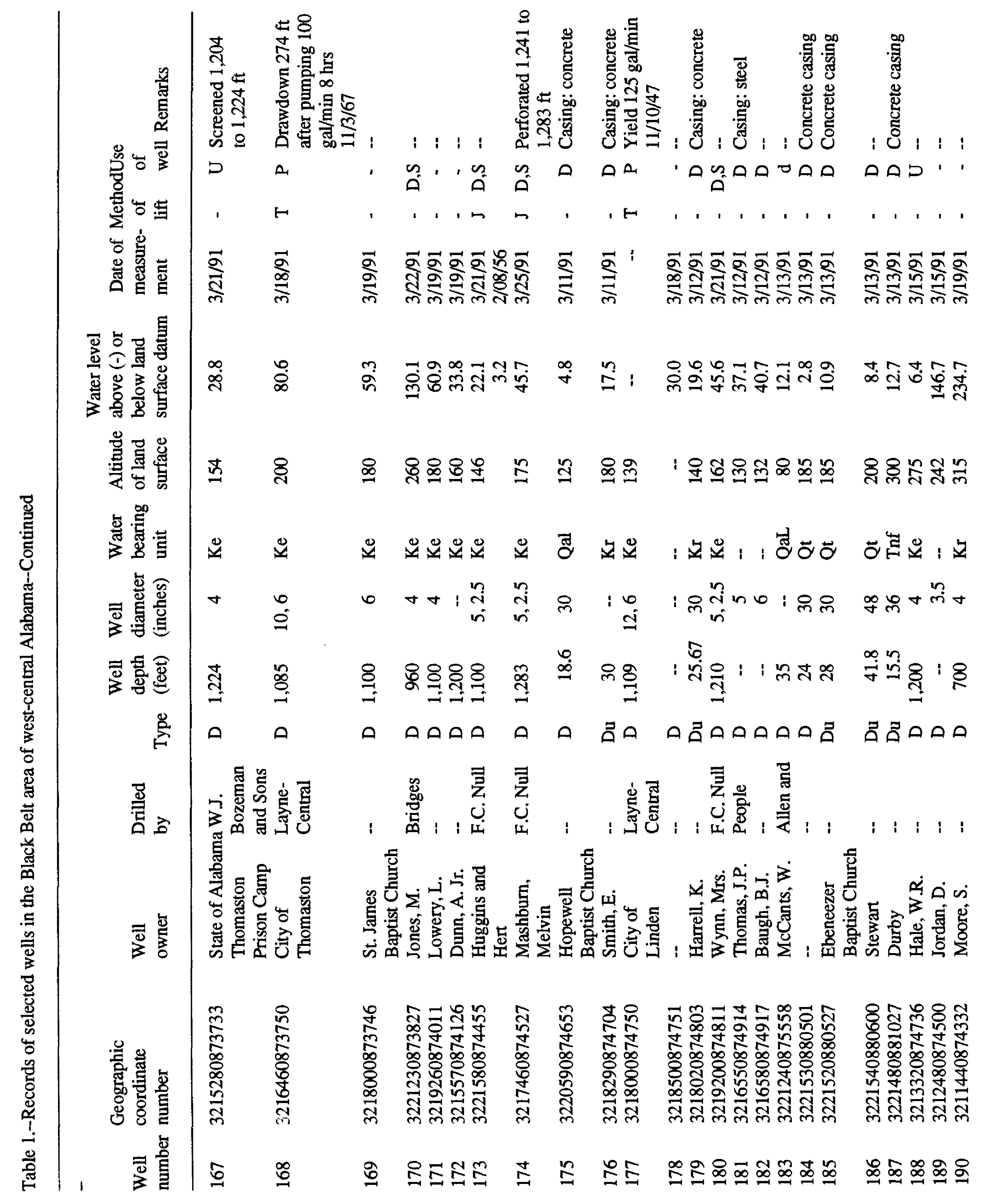




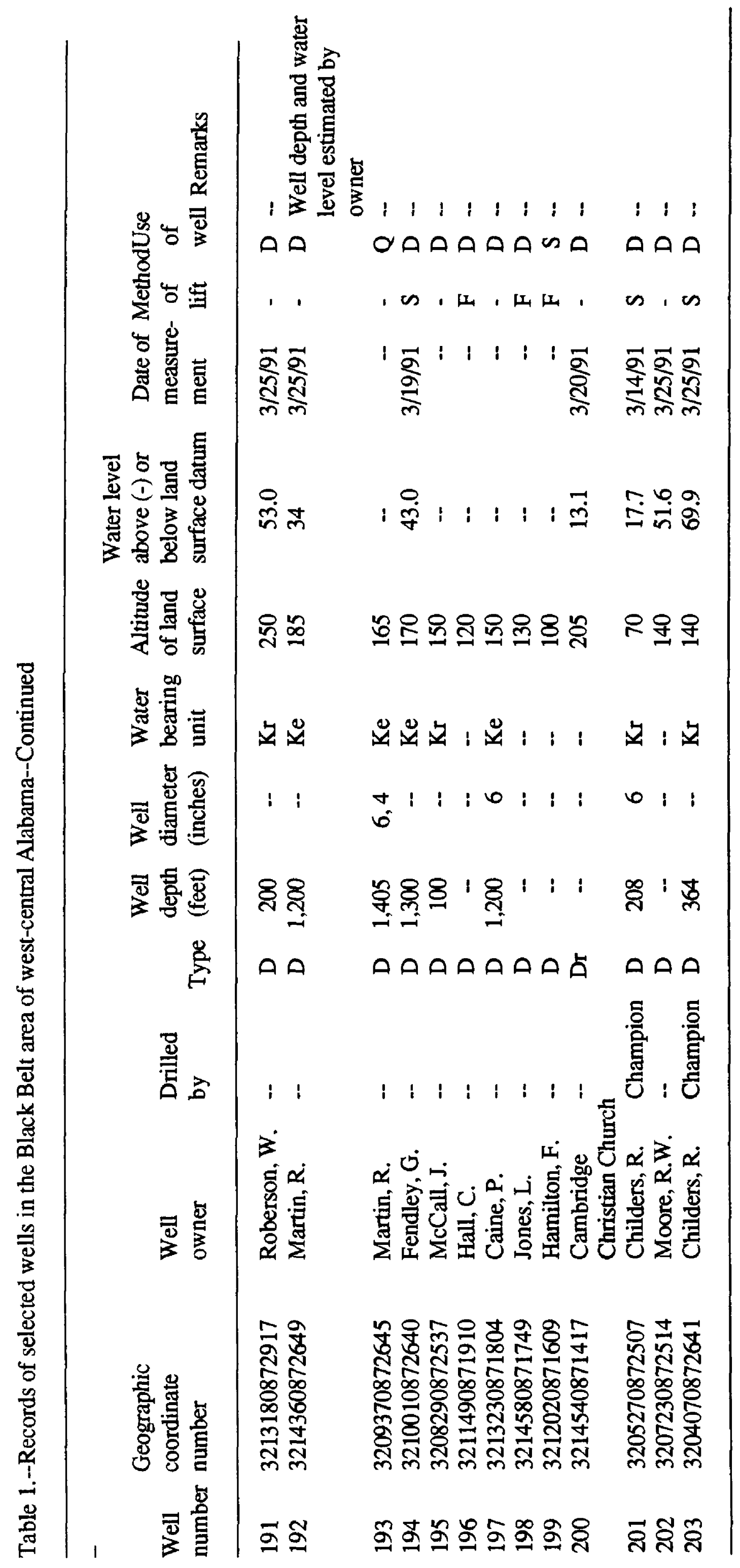




\section{Deposits of Tertiary Age}

Deposits of Tertiary age overlie the Cretaceous sediments and crop out across the extreme southern part of the study area (figs. 3 and 5). These deposits consist of sand, silt, clay, marl, limestone, conglomerate, and lignite. Deposits of Tertiary age in the study area consist of the Clayton and Porters Creek Formations, undifferentiated, the Naheola Formation, and the Nanafalia Formation of the Wilcox Group. All of these formations are of Paleocene age. None of these formations are major aquifers in the study area.

\section{Nanafalia Formation}

The Nanafalia Formation crops out only in small areas in Sumter County in the extreme southwestern corner of the study area (fig. 3). The Nanafalia overlies the Naheola and has a thickness of about 150 to 200 feet. The formation consists of sand, sandy marl, sandy clay, and lignite. The Nanafalia is tapped for public supplies south of the study area; however, within the study area, the limited occurrence of this unit precludes its use as a ground-water source.

\section{Naheola Formation}

The Naheola Formation crops out in a narrow band in the southwestern corner of the study area in Sumter County, and in a small area at the extreme southern part of the study area in Marengo County (fig. 3). The Naheola Formation has been subdivided into two members, Oak Hill Member (lower part) and the Coal Bluff Marl Member (upper part). The Oak Hill Member consists of laminated, silty clay containing interbeds of fine-grained sand and a thin bed of lignite near the top and generally is about 80 to 150 feet thick. The Coal Bluff Marl Member consists of laminated sand and clay, and is about 20 to 40 feet thick.

The Naheola Formation is not a ground-water source of any consequence in the study area, however, south of the study area it yields water for municipal and domestic supplies. Wells tapping the formation south of the study area reportedly produce as much as $40 \mathrm{gal} / \mathrm{min}$.

\section{Clayton and Porters Creek Formations}

The Clayton Formation overlies the Prairie Bluff Chalk in Sumter, Marengo, and Wilcox Counties and is, in turn, overlain by the Porters Creek Formation (fig. 3). The Clayton Formation consists principally of chalky marl, limestone, and conglomerate, although a thin basal sand bed is commonly present in this unit. The overlying Porters Creek Formation consists of massive marine clays. The combined thickness of the Clayton and Porters Creek Formations is 270 to 520 feet in the study area. The Clayton yields less than $10 \mathrm{gal} / \mathrm{min}$ to shallow dug wells in some outcrop areas. The Porters Creek is relatively impermeable and not a ground-water source. 


\section{Deposits of Cretaceous Age}

Cretaceous deposits crop out from the northern boundary of the study area to the southern parts of Marengo and Sumter Counties and the northern part of Wilcox County (fig. 3). These sediments strike northwestward and dip southwestward at 30 to $50 \mathrm{ft} / \mathrm{mi}$ (feet per mile). A subsurface profile of the southwestward-dipping units of Cretaceous age is shown in the diagrammatic geologic section in figure 5. Upper Cretaceous deposits include the major aquifers in the study area. These aquifers consist of beds of sand in the Coker, Gordo, and Eutaw Formations. Sands in the Ripley Formation, also in the Upper Cretaceous, are a minor aquifer in the study area. Because of the availability of water from shallower aquifers, Lower Cretaceous rocks are seldom tapped by wells in Alabama. However, interpretation of electric logs, sample cuttings, and analyses of water from test wells indicate that sand beds in the unit are very permeable and contain freshwater in updip areas. The water in these strata becomes progressively more saline downdip.

\section{Prairie Bluff Chalk}

The Prairie Bluff Chalk overlies the Ripley Formation, and crops out immediately south of, and parallel to, the Ripley (fig. 3). The Prairie Bluff is about 50 to 100 feet thick, and consists mainly of massive compact chalk and calcareous sandy clay. The Prairie Bluff is relatively impermeable and is not a ground-water source in the study area. It is a confining unit between the Clayton and Ripley aquifers.

\section{Ripley Formation}

The Ripley Formation overlies the Demopolis Chalk and crops out in the study area from west-central Sumter County across central Marengo, southern Perry, Dallas, and northern Wilcox Counties (fig. 3). The Ripley consists of beds of calcareous sandstone, sandy chalk, sand, sandy calcareous clay, and thin beds of fossiliferous sandstone. The thickness of the formation ranges from 35 to 300 feet.

The Ripley aquifer, which consists of sand beds in the Ripley Formation, is a minor groundwater source in the study area. Data for eight wells tapping the Ripley are given in table 1. Downdip, south of the study area, the Ripley is a source of public water supply, but in the study area wells tapping the Ripley generally yield $10 \mathrm{gal} / \mathrm{min}$ or less. 


\section{Demopolis Chalk}

The Demopolis Chalk overlies the Mooreville Chalk, and crops out in a belt as much as 8 miles wide in northeastern Sumter County (fig. 3). The formation consists mainly of silty micaceous fossiliferous chalk. The basal beds are overlain by nearly pure chalk which contains 75 to 90 percent calcium carbonate. The thickness of the formation ranges from 200 to 500 feet. The Demopolis is not a ground-water source in the study area.

\section{Mooreville Chalk}

The Mooreville Chalk crops out parallel to and immediately south of the Eutaw Formation. The outcrop extends from southwestern Pickens County southeastward across the study area in central Dallas County (fig. 3). The Mooreville consists of silty chalk and calcareous clay interbedded with thin layers of limestone and calcareous sandstone. The thickness of the Mooreville ranges from 350 feet in Sumter County to about 420 feet in Dallas County. The Mooreville is not a ground-water source in the study area, but, together with the Demopolis Chalk, forms a confining unit for the underlying Eutaw aquifer.

\section{Eutaw Formation}

The Eutaw Formation overlies the Gordo Formation and crops out across central Hale, Perry, and northern Greene Counties (fig. 3). The thickness of the Eutaw in the study area is about 400 feet. The lower part of the formation consists of thin to massive beds of fine- to coarsegrained sand with interbedded clay. The middle part consists chiefly of fine- to medium-grained, well sorted sand with interbedded clay. The upper part of the formation consists of about 100 feet of very fine-grained sand locally containing layers of sandstone and chalky sand.

Sand and gravel beds in the upper and lower parts of the Eutaw Formation constitute the Eutaw aquifer. The Eutaw aquifer is the most extensively used ground-water source in the study area (table 1; plate 2). Data from 116 wells tapping the Eutaw are given in table 1. Most of the public water systems in the study area pump water from the sand beds of this aquifer. Mooty (1987) reported that wells developed in the Eutaw aquifer are capable of producing as much as $1,500 \mathrm{gal} / \mathrm{min}$. Gardner (1981) used transmissivities of $2,200 \mathrm{ft}^{2} / \mathrm{d}$ (square feet per day) to 5,800 $\mathrm{ft}^{2} / \mathrm{d}$ in simulating the ground-water flow system of the Eutaw aquifer in west-central Alabama. Planert and Sparkes (1985) calculated the transmissivity of the Eutaw to be $2,000 \mathrm{ft}^{2} / \mathrm{d}$ in northeastern Marengo County. 


\section{Gordo Formation}

The Gordo Formation overlies the Coker Formation and crops out in northern Perry County and north of the study area in a northwestward trending belt (figs. 3 and 4). The formation consists of 300 to 450 feet of nonmarine lenticular beds of gravel, sand, and clay (fig. 6). The Gordo can be distinguished from the underlying Coker by its darker and more reddish sands and purple mottled clays. Poorly-sorted, coarse-grained sand and gravel are prevalent in the lower part of the formation. The upper part of the formation consists of lenticular clay and fine- to coarse-grained sand. The sand and gravel beds in the Gordo Formation constitute the Gordo aquifer.

The Gordo aquifer is one of the major aquifers in the study area. It is a principal source of water for Greensboro, Marion, Faunsdale, Union, North Dallas County, and the Dallas County public water supply systems. Data for wells tapping the Gordo aquifer are given in table 1. The Gordo aquifer is used as a source of water as far south as northeastern Marengo County.

Two municipal wells at Greensboro have specific capacities of 36 and $24 \mathrm{gal} / \mathrm{min} / \mathrm{ft}$ (gallons per minute per foot) of drawdown. Based on these data, it is estimated that wells of $1,000 \mathrm{gal} / \mathrm{min}$ or more could be constructed in the Gordo aquifer. Gardner (1981) used transmissivities of 2,600 $\mathrm{ft}^{2} / \mathrm{d}$ to $8,100 \mathrm{ft}^{2} / \mathrm{d}$ in simulating the ground-water flow system of the Gordo aquifer in westcentral Alabama. Planert and Sparkes (1985) estimated the transmissivity of the Gordo to be $10,000 \mathrm{ft}^{2} / \mathrm{d}$ in northeastern Marengo County.

\section{Coker Formation}

The Coker Formation is the basal unit of the Upper Cretaceous Series in Alabama, and is present in the subsurface throughout the study area. The thickness of the formation ranges from 500 to 1,000 feet. The Coker consists of a basal nonmarine zone of sand, gravel, and clay that was deposited in a deltaic environment. The coarser sand beds and beds of gravel are near the bottom of the formation. The sands generally become progressively finer grained and less abundant toward the top of the formation where clay predominates. However, in Sumter County mediumto coarse-grained sand and gravel beds are present in the upper part of the Coker in some places. In Hale County, a clay zone is a confining unit between the Coker aquifer and the overlying Gordo aquifer. In Dallas County, the upper part of the formation may include a lenticular bed of fine- to medium-grained sand, which is of hydrologic importance for water supplies. Sand and gravel beds in the formation comprise the Coker aquifer (fig. 6).

The Coker aquifer is little used in the study area because of the excessive depth of the aquifer and the availability of water in the shallower Gordo and Eutaw aquifers. Three wells inventoried in the study area produce water from the Coker aquifer. These are the public-supply wells at Akron (well 12 in table 1), the well owned by the North Sumter County Water Authority (well 1 in table 1), and a well in central Greene County (well 53 in table 1). The North Sumter 
County Water Authority well is located about 2.5 miles south of Geiger on Alabama Highway 17, and is the most downdip Coker well in Alabama (well 1 in table 1). This well is screened from 1,912 to 1,982 feet.

Data are insufficient to evaluate the hydraulic characteristics of the Coker aquifer throughout the study area. However, based on an aquifer test conducted June 12-13, 1991, at the North Sumter County Water Authority well, the Coker had a transmissivity of $5,000 \mathrm{ft}^{2} / \mathrm{d}^{2}$ and a potentiometric head of 34.4 feet above land surface.

\section{Lower Cretaceous Rocks}

Lower Cretaceous rocks underlying the Coker Formation are not a major source of water in the study area, because of the depth of these rocks and elevated chloride concentrations in water from these deposits. One water-supply well for 14 catfish ponds in west-central Hale County is screened at a depth of 1,410 feet in a water-bearing unit that could be in Lower Cretaceous rocks. This well flowed at an estimated $2,500 \mathrm{gal} / \mathrm{min}$ in 1922, but by 1968 the rate of flow had decreased to $900 \mathrm{gal} / \mathrm{min}$. Water from this well had a chloride concentration of more than 2,400 $\mathrm{mg} / \mathrm{L}$ in 1991.

\section{Recharge to and Discharge from the Major Aquifers}

The major aquifers in the study area are sand and gravel beds in the Eutaw, Gordo, and Coker Formations (fig. 6). Water in these aquifers occurs under artesian conditions in most of the study area. Water enters the aquifers in topographically high outcrop areas, flows laterally along short flow paths to discharge into streams or downdip into the confined parts of the aquifers (Miller, 1992).

\section{Recharge}

Ground-water recharge is the replenishment of ground water by downward infiltration of water from rainfall, streams, and other sources (American Society of Civil Engineers, 1987, p. 222). In the study area, rainfall is the primary source of recharge. The rainfall that percolates through the soil to the water table is ground-water recharge, and the areas where accretions to ground water occur are called recharge areas.

Recharge areas for the major aquifers in the study area generally are congruent with the outcrops of the Eutaw, Gordo, and Coker Formations (fig. 4). Most of the recharge area of the Gordo aquifer and all of the recharge area of the Coker aquifer are north of the study area (DeJarnette and Crownover, 1987; Mooty, 1987). 
Ground water moves from recharge areas to downgradient discharge areas, such as stream valleys, swamps, lakes, or pumping centers. Water can also move from one aquifer to another through intervening low-permeability material, called confining beds, into an underlying aquifer. Gardner (1981) concluded that large amounts of water in the Eutaw and Gordo aquifers are leaking upward through confining layers to discharge in Alabama, Black Warrior and Tombigbee River Valleys.

Ground water that moves from recharge areas and discharges to streams sustains the flow of the streams (baseflow), especially during periods of no rainfall. Although this water is considered to be surface runoff from the drainage basin, it is derived from ground-water storage. One of the most widely used methods for determining ground-water recharge rates in the humid eastern United States involves analysis of streamflow data to determine baseflow (Vechiolli and others, 1990). Assuming there is no long-term change in the amount of ground water in storage, groundwater discharge, as measured by the baseflow of a stream, approximately equals the amount of ground-water recharge to the aquifer underlying the basin. Other discharge from the ground-water reservoir, such as withdrawals by wells or evapotranspiration from the water table, need to be considered in estimating recharge.

Methods developed by Rutledge $(1991,1992)$ were used to estimate recharge to the major aquifers in the study area. The methods used were calculation of the master recession curve and streamflow partitioning. The calculation of the master recession curve is requisite to the streamflow partitioning method of streamflow analysis. An empirical method is used to obtain from streamflow record a nonlinear master recession curve, which is a mathematical expression for streamflow recession during times of negligible direct runoff. The method is based on the assumption that the storage delay factor, which is the time per log cycle streamflow of recession, varies linearly with the logarithm of streamflow. In this method, the analyst uses a computer program to read a data file of daily mean streamflow and can select segments of streamflow recession curves for analysis.

The streamflow partitioning method can be used to estimate a continuous record of ground water discharge, or baseflow, from the streamflow hydrograph. The integration of this record over a period of several years provides an estimate of recharge. This method is based on the assumption that ground-water discharge is equal to streamflow during periods of negligible surface runoff; and that ground-water discharge between these periods can be interpolated.

Streamflow data from eight daily-record sites north of the study area were analyzed (fig. 1). These sites are on unregulated streams in the outcrop areas of the major aquifers and the period of record for each site was 10 years or more. Recharge determined from streamflow partitioning ranged from $7.0 \mathrm{in} / \mathrm{yr}$ at Fivemile Creek near Greensboro to $14.3 \mathrm{in} / \mathrm{yr}$ at Sipsey River near Pleasant Ridge (table 2). The average recharge to the major aquifers was estimated to be 
Table 2.--Recharge to the major aquifers in the Black Belt area of west-central Alabama, estimated from baseflow of streams at selected gaging stations

[Map number corresponds to those on figure 1. Major aquifer outcrop: 1, Coker; 2, Gordo; 3, Eutaw (listed in order of descreasing outcrop area). $\mathrm{mi}^{2}$, square mile; in/yr, inches per year.]

\begin{tabular}{|c|c|c|c|c|c|c|}
\hline $\begin{array}{l}\text { Map } \\
\text { number }\end{array}$ & $\begin{array}{l}\text { Station } \\
\text { number }\end{array}$ & Station name & $\begin{array}{l}\text { Major } \\
\text { aquifer } \\
\text { outcrop }\end{array}$ & $\begin{array}{l}\text { Drainage } \\
\text { area } \\
\left(\mathrm{mi}^{2}\right)\end{array}$ & $\begin{array}{l}\text { Time } \\
\text { period } \\
\text { analyzed } \\
\text { (years) }\end{array}$ & $\begin{array}{l}\text { Estimated } \\
\text { recharge } \\
\text { (in/yr) }\end{array}$ \\
\hline 1 & 02442000 & Luxapallila Creek near Fayette & 1 & 130 & $1945-70$ & 12.7 \\
\hline 2 & 02445500 & Sipsey River at Fayette & 1 & 282 & $1939-59$ & 10.2 \\
\hline 3 & 02444000 & Coal Fire Creek near Pickensville & 1 & 126 & $1955-80$ & 11.9 \\
\hline 4 & 02445000 & Lubbub Creek near Carrollton & 2,3 & 112 & 1954-64 & 8.6 \\
\hline 5 & 02446500 & Sipsey River near Elrod & 1 & 528 & $1928-90$ & 14.0 \\
\hline 6 & 02447000 & Sipsey River near Pleasant Ridge & $3,2,1$ & 769 & $1939-59$ & 14.3 \\
\hline 7 & 02465493 & Elliotts Creek at Moundville & 3,2 & 32 & $1977-90$ & 12.4 \\
\hline 8 & 02465500 & Fivemile Creek near Greensboro & 3,2 & 73 & $1955-71$ & 7.0 \\
\hline
\end{tabular}


$11.4 \mathrm{in} / \mathrm{yr}$. It should be noted that recharge generally decreases downdip of the aquifer outcrop areas (Miller, 1992).

The recharge estimates are based on the outcrop areas of the major aquifers excluding those areas overlain by alluvial deposits, which are considered drainage areas. Recharge rates on the order of $11 \mathrm{in} / \mathrm{yr}$ seem reasonable because the surficial sediments in the outcrop areas of the Eutaw, Gordo, and Coker Formations were estimated from geophysical logs to be about half sand and half clay. Davis and others (1975) also determined that these formations were 50 percent sand in Hale County. The recharge area of the Eutaw aquifer is in Pickens, Greene, Hale, Perry, Autauga, Chilton, and Dallas Counties, and covers an area of about $600 \mathrm{mi}^{2}$ (fig. 4). The recharge area of the Gordo aquifer is mainly in Pickens, Tuscaloosa, Hale, Bibb, Autauga, Perry, and Chilton Counties, and covers an area of about $300 \mathrm{mi}^{2}$. The recharge area of the Coker aquifer is mainly in Tuscaloosa, Bibb, and Chilton Counties, and covers an area of about $400 \mathrm{mi}^{2}$. Based on the above information, the recharge values for the major aquifers were estimated to be $340 \mathrm{Mgal} / \mathrm{d}$ for the Eutaw aquifer; $170 \mathrm{Mgal} / \mathrm{d}$ for the Gordo; and $230 \mathrm{Mgal} / \mathrm{d}$ for the Coker.

\section{Natural Discharge and Ground-Water Withdrawals}

Discharge from the major aquifers in the study area includes natural discharge through seeps and springs, which provide the base (dry weather) flow of streams and ground-water withdrawals from wells. Discharge to the streams and rivers is enhanced where the channels are deeply entrenched into the aquifers. Discharge to streams can occur by upward leakage through the lowpermeability confining unit or through fractures in consolidated confining units, such as the Mooreville and Demopolis Chalks (Gardner, 1981). Most of the remainder of the discharge is through wells. Wells are used for domestic, stock, industrial, aquacultural, public water supply, and irrigation purposes. A substantial amount of water is lost from the aquifer through flowing artesian wells. Based on previous reports and data collected during this study, an estimated 20.7 $\mathrm{Mgal} / \mathrm{d}$ discharges through flowing wells in the study area. Many wells in the study area that formerly flowed have ceased to flow in recent years as a result of lowering of the potentiometric surfaces of the aquifers. The largest pumping centers in the study area are near the cities and town that use ground water for public supplies. The total ground-water withdrawals in the study area and in the aquifer recharge areas to the north were about $72.8 \mathrm{Mgal} / \mathrm{d}$ in 1990 (Baker and Mooty, 1993).

The steady decline in the potentiometric surface of the Eutaw aquifer is indicated by hydrographs of water levels in selected wells screened in the Eutaw (fig. 7). Local depressions in the potentiometric surface of the aquifers were indicated in studies by DeJarnette and Crownover (1987), Mooty (1987), and Gardner (1981). These depressions generally coincide with natural 


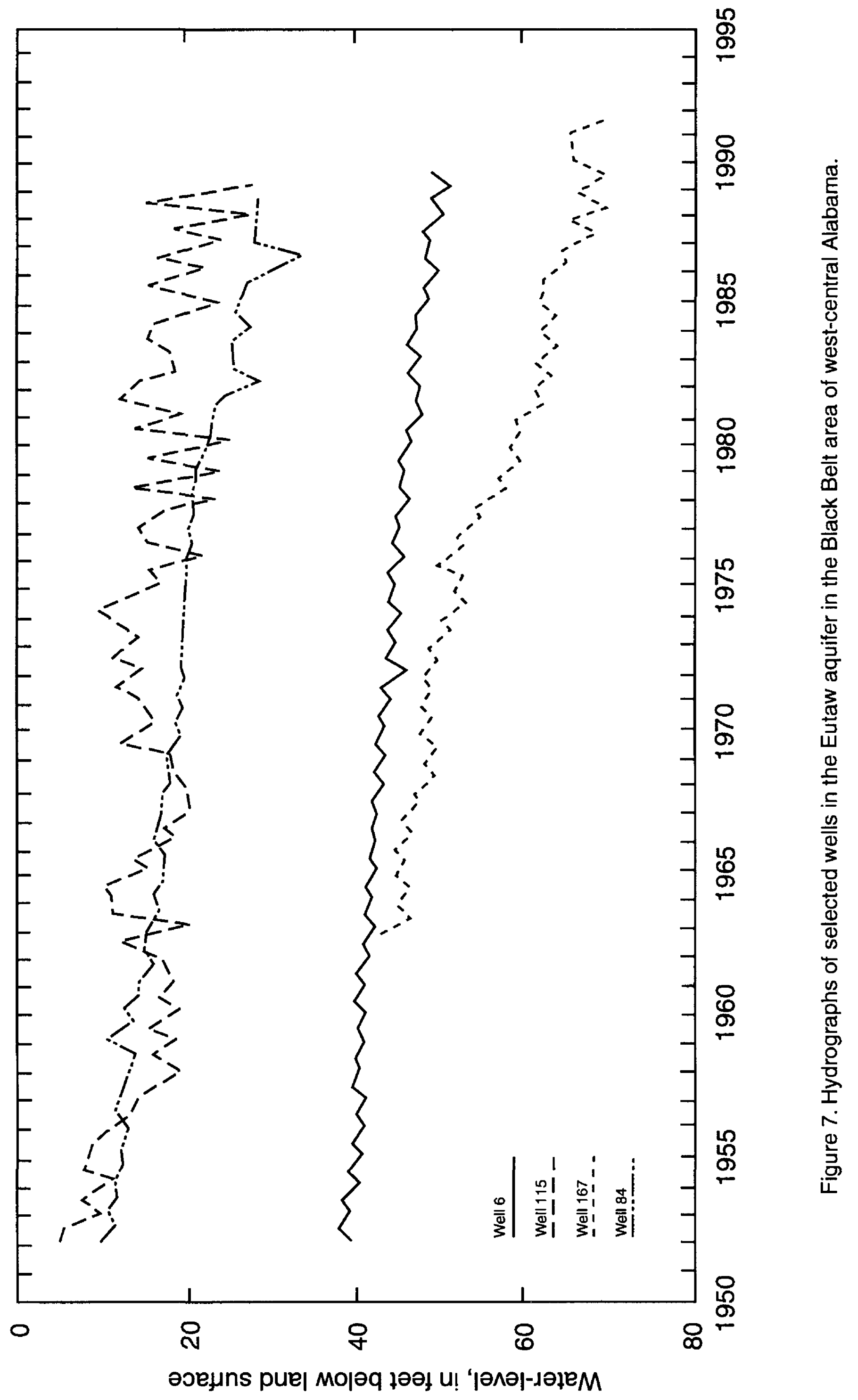


discharge areas along major rivers and major pumping centers near the larger cities and towns in and near the study area. Depressions have formed in the potentiometric surfaces of the Eutaw, Gordo, and Coker aquifers at the city of Demopolis (Williams and others, 1986a, 1986b) and in the potentiometric surface of the Eutaw aquifer at Marion (Williams and others, 1986b).

\section{GROUND-WATER QUALITY}

The chemical quality of ground water may limit its usefulness for particular purposes. Water used for municipal supplies generally must meet drinking water standards that provide for maximum chloride and dissolved-solids concentrations of 250 and $500 \mathrm{mg} / \mathrm{L}$, respectively. Quality requirements for industrial water depend on the type of use made of the water. Some industries have quality requirements that are far more exacting than requirements for municipal supplies; other industries, such as aquaculture, can use moderately mineralized water. Domestic water users are usually concerned with the hardness of water and with iron and chloride content of the water. Hard water is objectionable for some uses because it increases soap consumption and may deposit scale in pipes and boilers; soft water under certain conditions may induce corrosion. General terms used in this report to describe hardness of water expressed as milligrams per liter of calcium carbonate are: soft, 0-60 mg/L; moderately hard, 61-120 mg/L; hard, 121-180 mg/L; and very hard, $181 \mathrm{mg} / \mathrm{L}$ or more. Iron in excess of $0.3 \mathrm{mg} / \mathrm{L}$ may cause staining of porcelain or enamel fixtures, clothing, or other fabrics.

Chloride concentration in water can affect the suitability of the water for many uses. If chloride is present in sufficient concentration (in excess of $1,000 \mathrm{mg} / \mathrm{L}$ ), the water has a salty taste. Water with chloride concentration of less than $250 \mathrm{mg} / \mathrm{L}$ generally is desired for domestic and municipal use; however, water with substantially higher chloride concentrations can be used for fish farming. Catfish farmers often add sodium chloride to catfish ponds to control or prevent fish disease. The dissolved chloride concentrations in water from wells sampled in the study area are shown on plate 3. Chloride concentrations in water from the Eutaw aquifers in central Greene County commonly exceed $500 \mathrm{mg} / \mathrm{L}$. Other areas of relatively high chloride concentrations in water from the Eutaw aquifer are in downdip areas in Marengo and Wilcox Counties.

The chemical characteristics of ground water depend on several variables, such as composition of the aquifer, distance from recharge areas, and the period of time water has been in contact with minerals in the aquifer. An evaluation of the results of chemical analyses of ground water in the study area indicates that the water is satisfactory for most uses. The results of chemical analyses of water samples collected from selected wells in the study area are given in table 3 . 


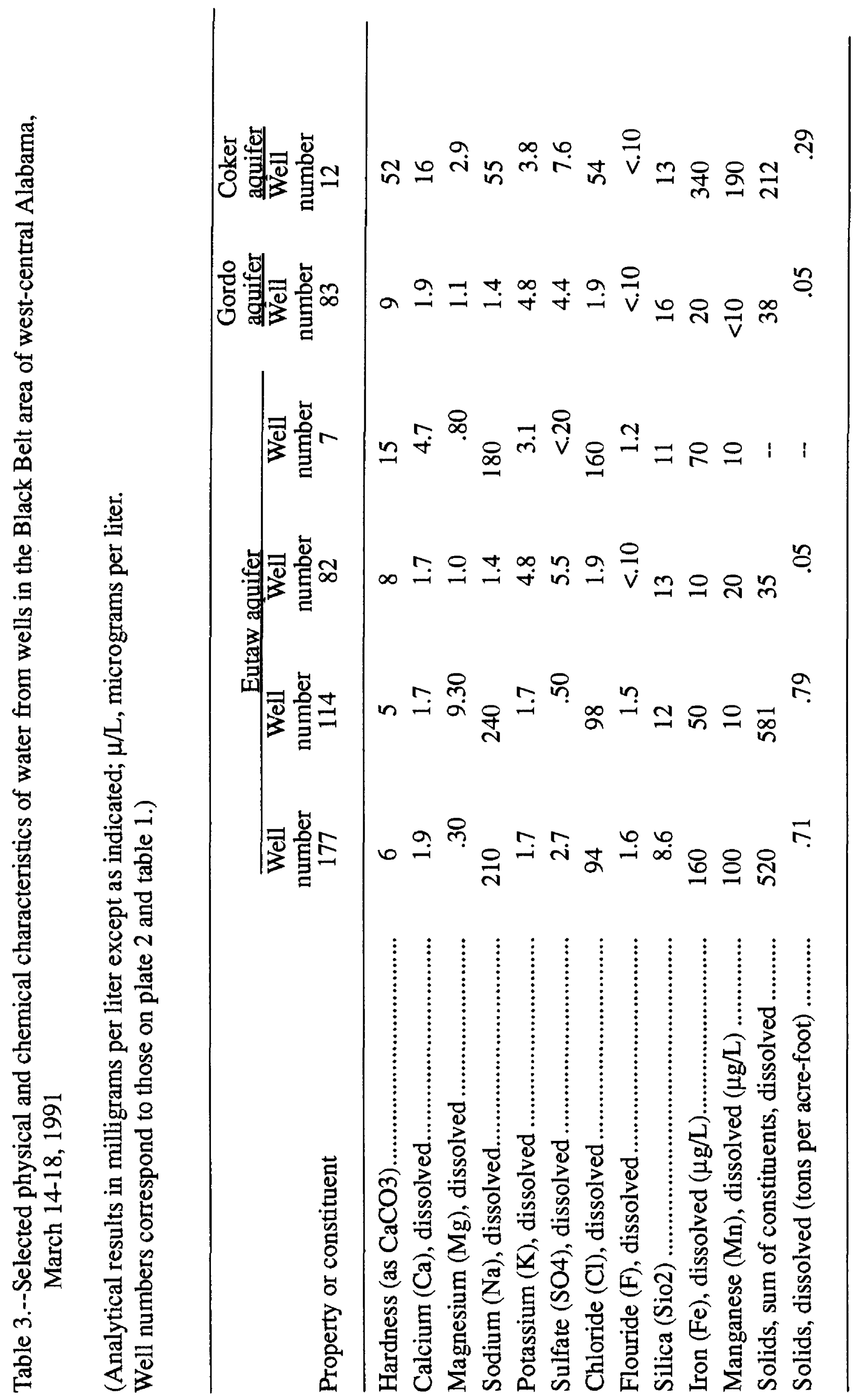


Water in the Eutaw aquifer in the study area generally is suitable for public water supply except in central Greene County, where chloride concentrations commonly exceed $500 \mathrm{mg} / \mathrm{L}$ (plate 3). Water samples from isolated wells completed in the Eutaw aquifer in some other parts of the study area have chloride concentrations that exceed $250 \mathrm{mg} / \mathrm{L}$ (plate 3); however, water from nearby wells completed in this aquifer have relatively low concentrations of chloride. Results of analyses of major constituents for water samples collected from four wells tapping the Eutaw aquifer are listed in table 3. Dissolved iron concentrations in these samples range from 10 to 160 $\mu \mathrm{g} / \mathrm{L}$ (micrograms per liter). Dissolved sodium concentrations in water samples from the Eutaw aquifer are higher than those in samples from the other major aquifers (table 3).

Water from the Gordo aquifer in the study area generally is of good quality and suitable for most uses. Water from four wells tapping the Gordo aquifer in the study area had a range of dissolved chloride concentrations from 1.6 to $630 \mathrm{mg} / \mathrm{L}$ (table 1; plate 3). The Greensboro Utility well (well 83 in tables 1 and 3 ) produces water from the Gordo aquifer and this water was among the least mineralized waters sampled.

The Coker aquifer is tapped by few wells in the study area; therefore, only limited data on the chemical quality of water in this aquifer are available. However, based on data from wells in Hale County in the northern part of the study area and the North Sumter County Water Authority well, water from the Coker Formation in the northern part of the study area generally is soft to moderately hard and can contain iron concentrations in excess of $0.3 \mathrm{mg} / \mathrm{L}$. Chemical analyses of water from the Sumter County well indicated that the chloride concentrations in water from this well were less than $25 \mathrm{mg} / \mathrm{L}$. Water-quality data for wells tapping the Coker aquifer north of the study area indicate that water from this aquifer has chloride concentrations ranging from 2 to 790 $\mathrm{mg} / \mathrm{L}$.

\section{WATER USE FOR AQUACULTURE, 1990}

Operational water-use requirements for aquaculture were calculated from data obtained from an inventory of catfish ponds. This inventory indicated that 1,118 ponds representing a total surface area of 15,647 acres were in operation in the study area in 1990 (table 4; plate 1). As used in this section of the report, surface water refers to water in flowing streams, and runoff refers to stormwater runoff from precipitation. More than 60 percent of the ponds inventoried depended on rainfall runoff as their only source of water. Less than 1 percent of the ponds depended on ground water as a sole source of water. Water-use data were calculated on a site-by-site basis, using information supplied by the pond owners or operators. Where specific information was unavailable for a particular site, estimates were made based on information from similar sites. 


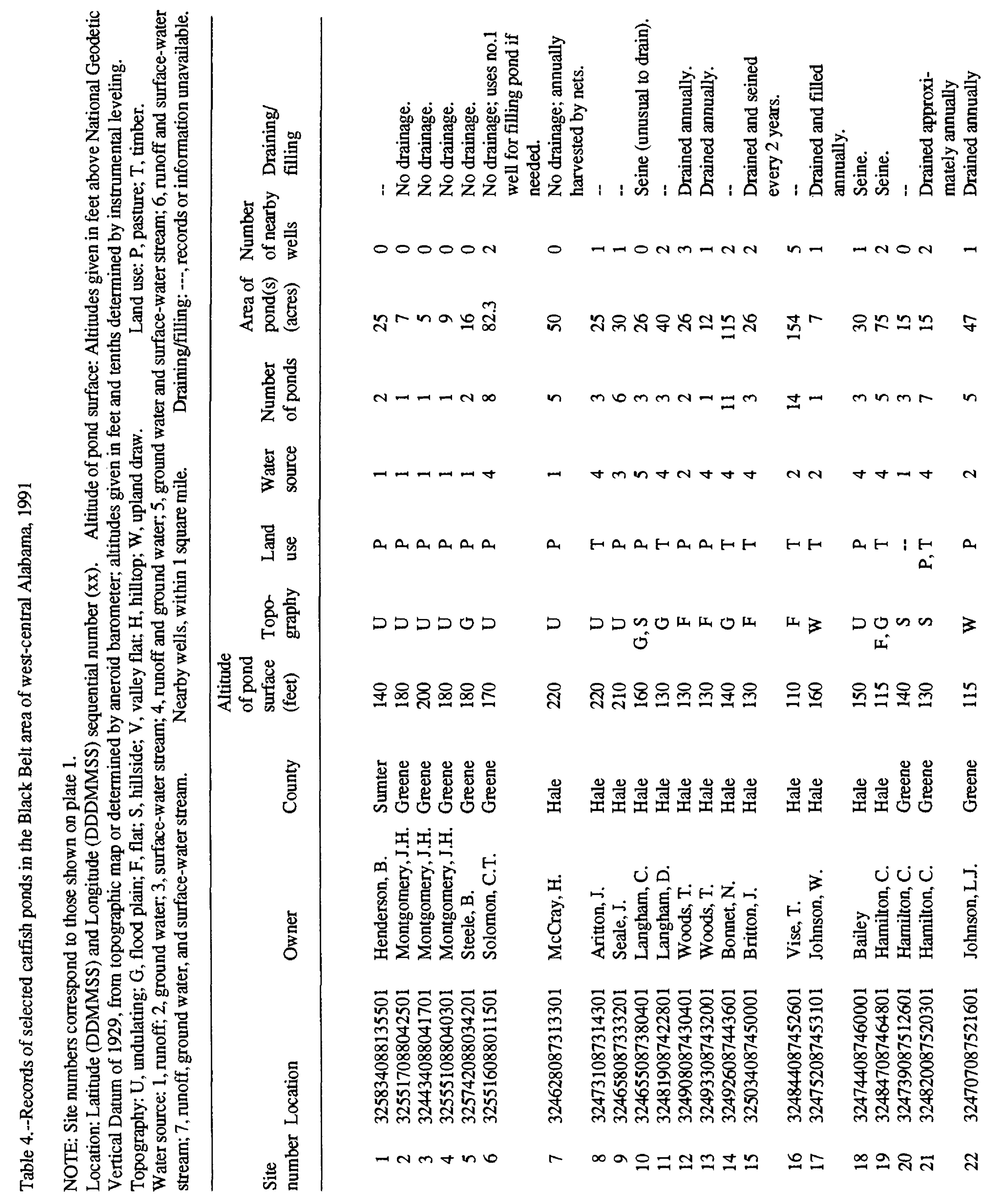




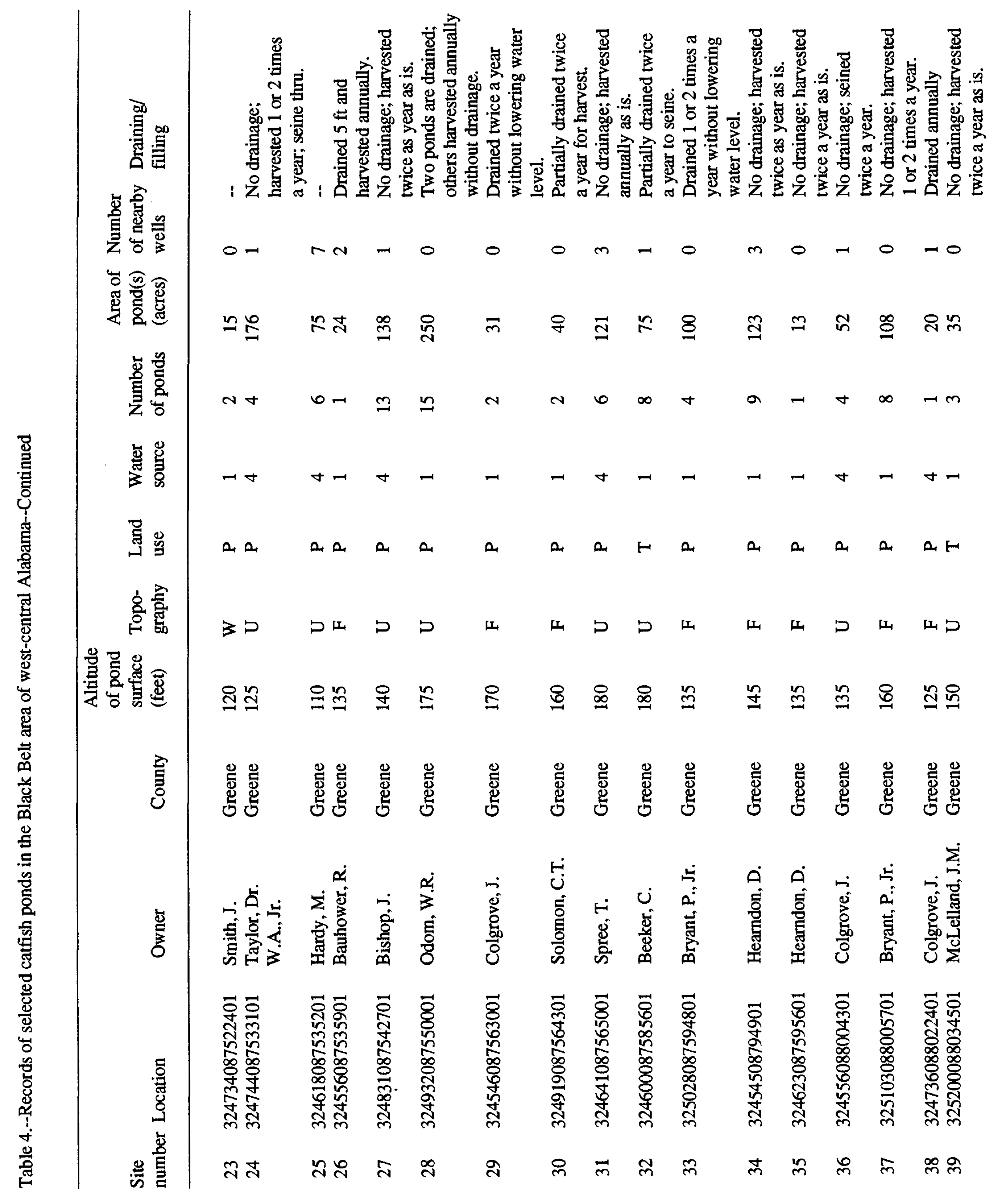




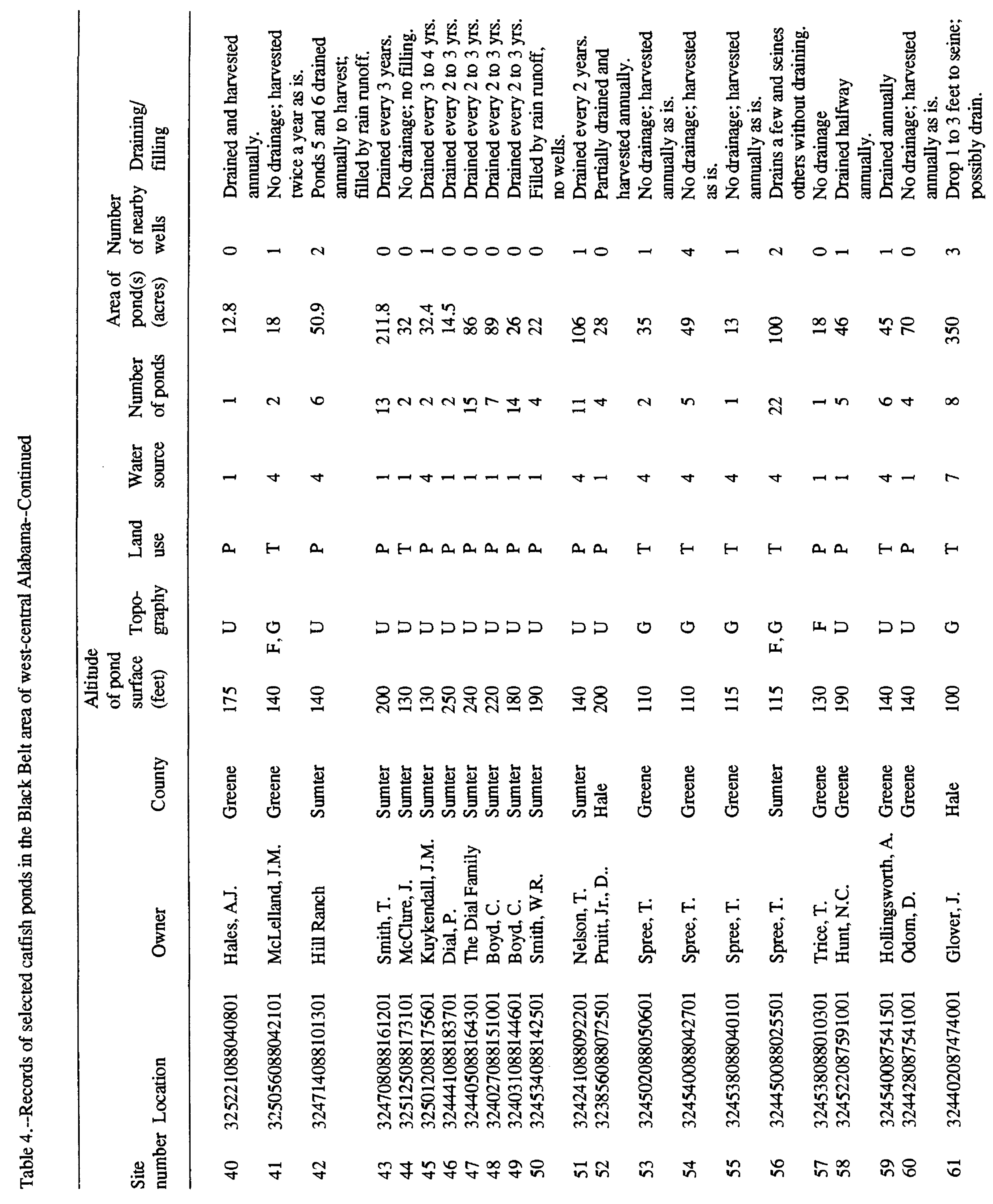




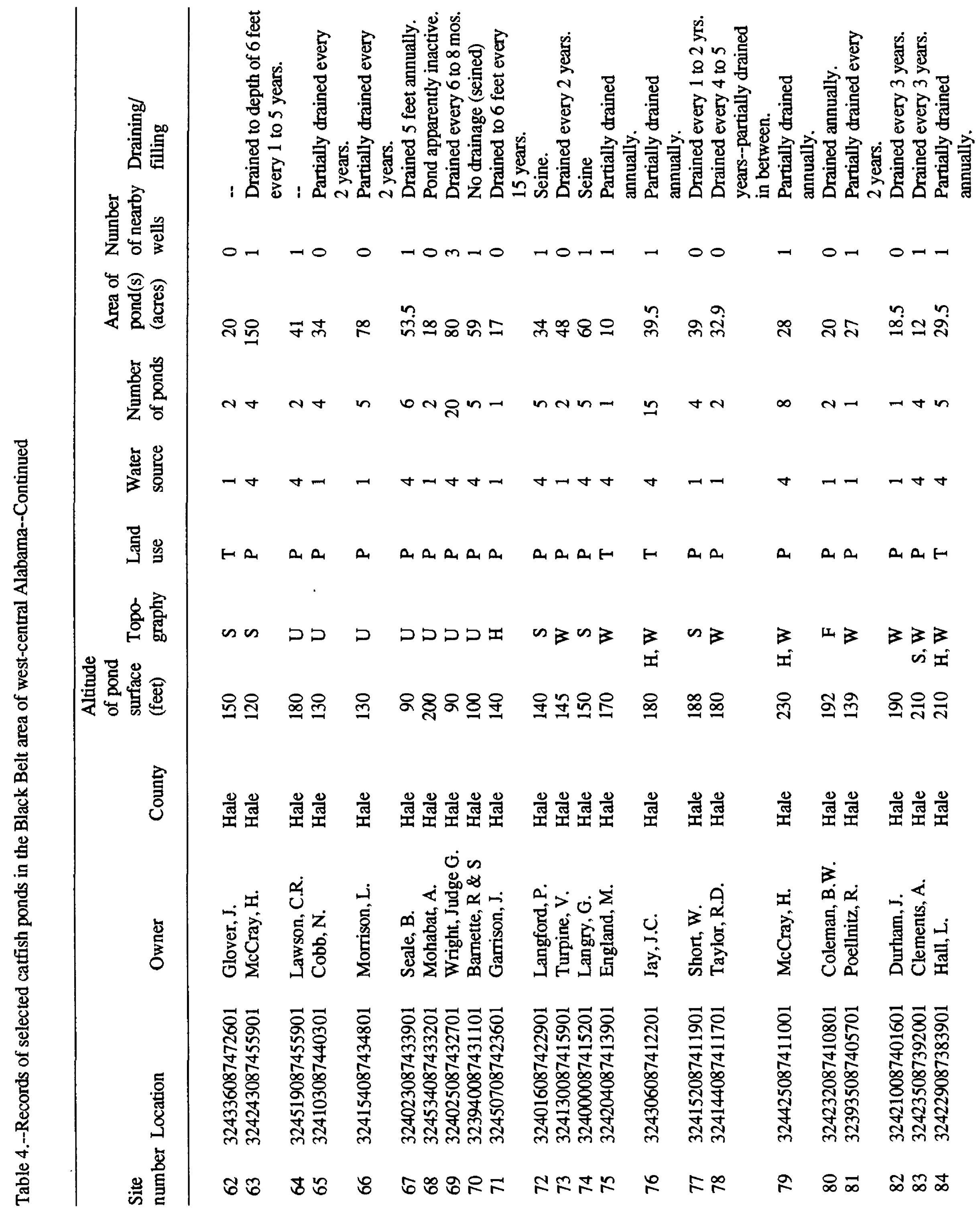




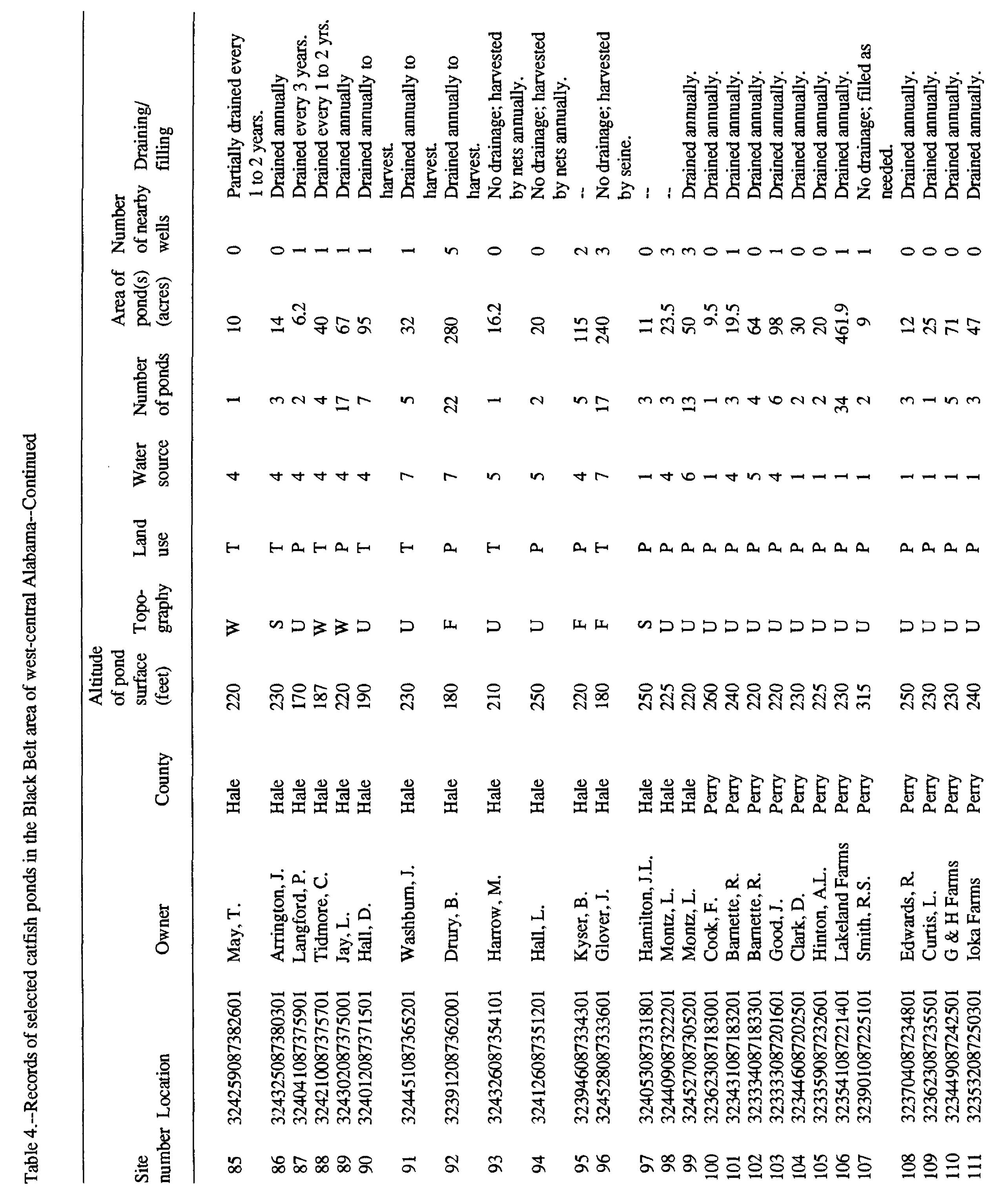




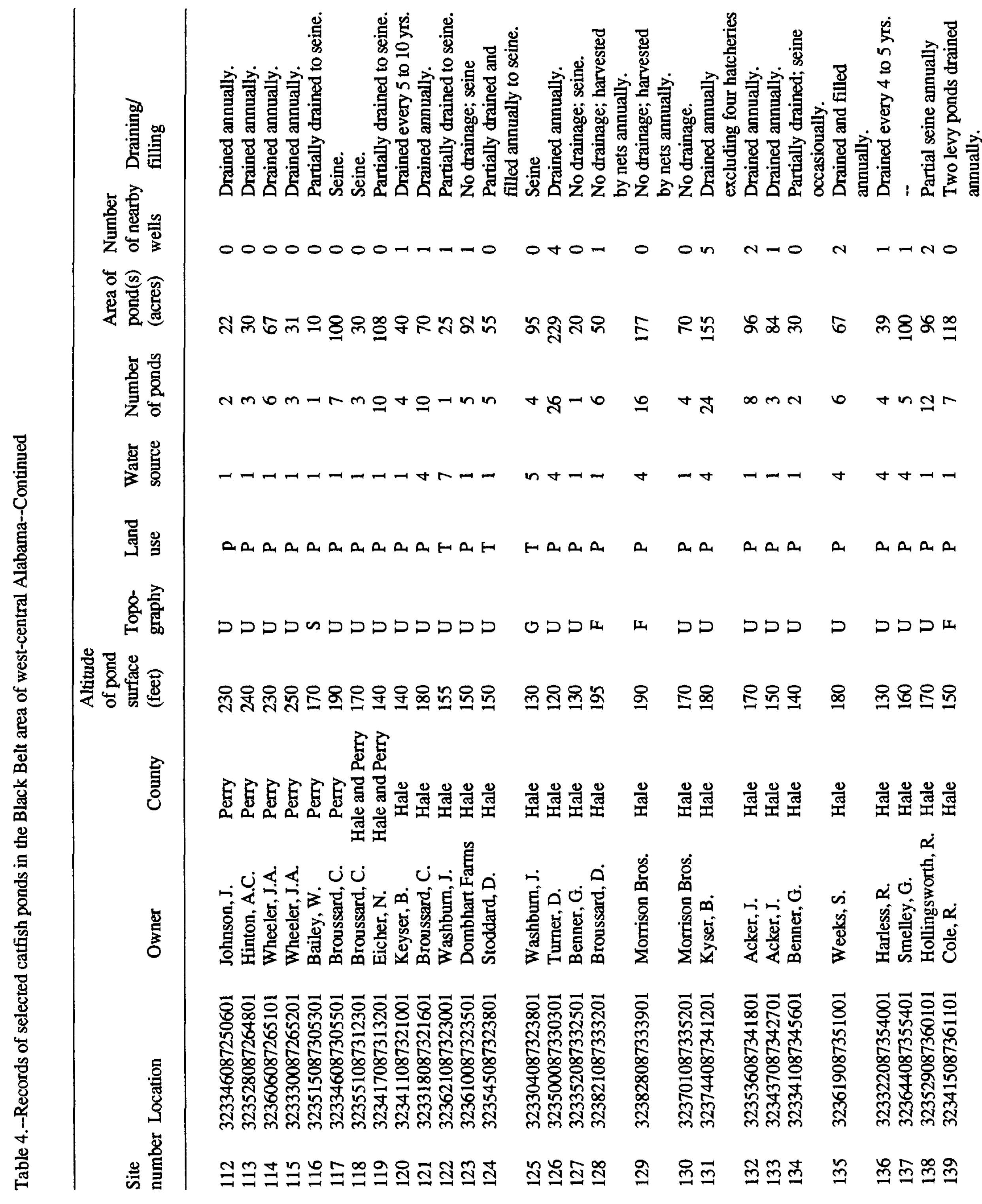




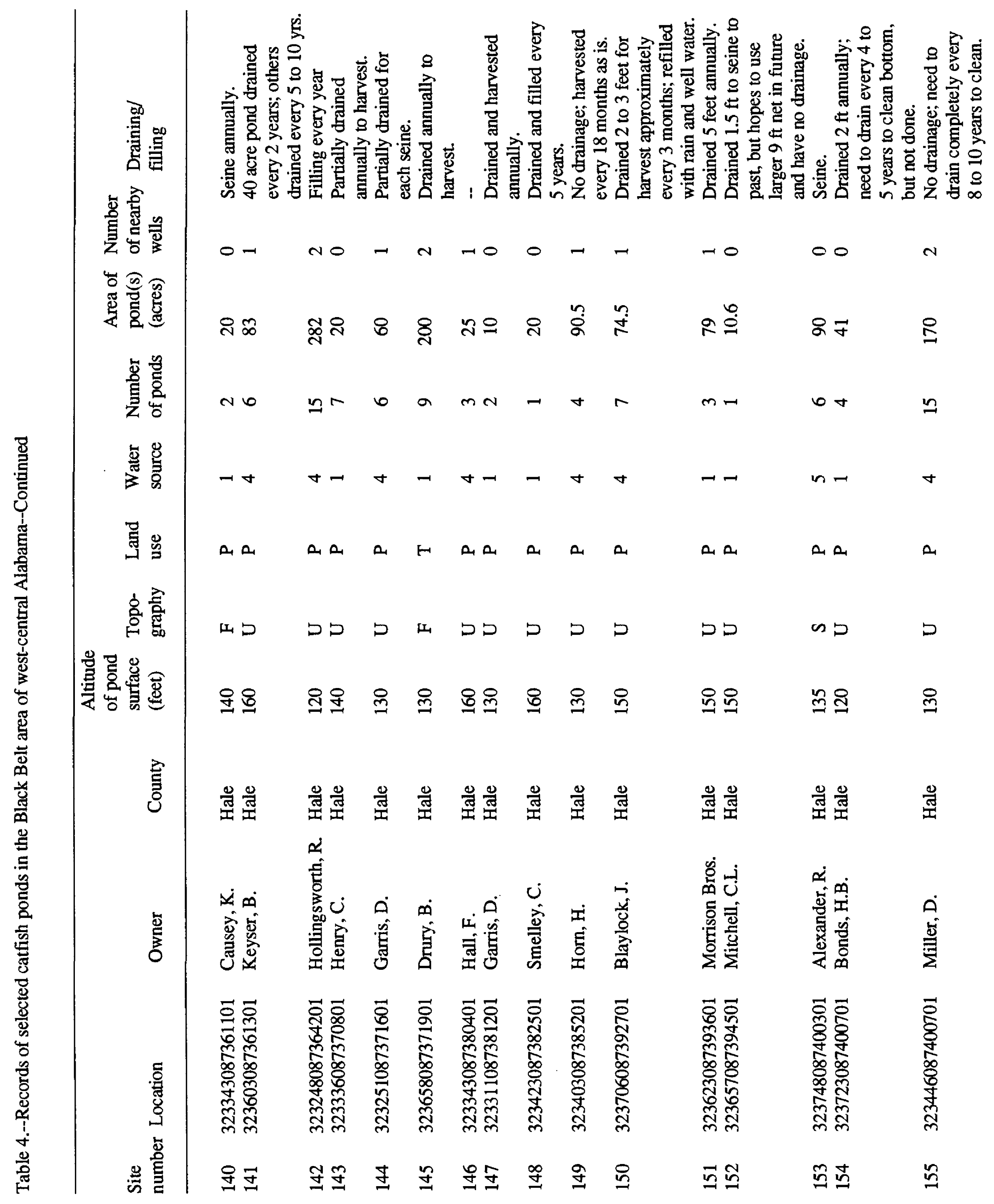




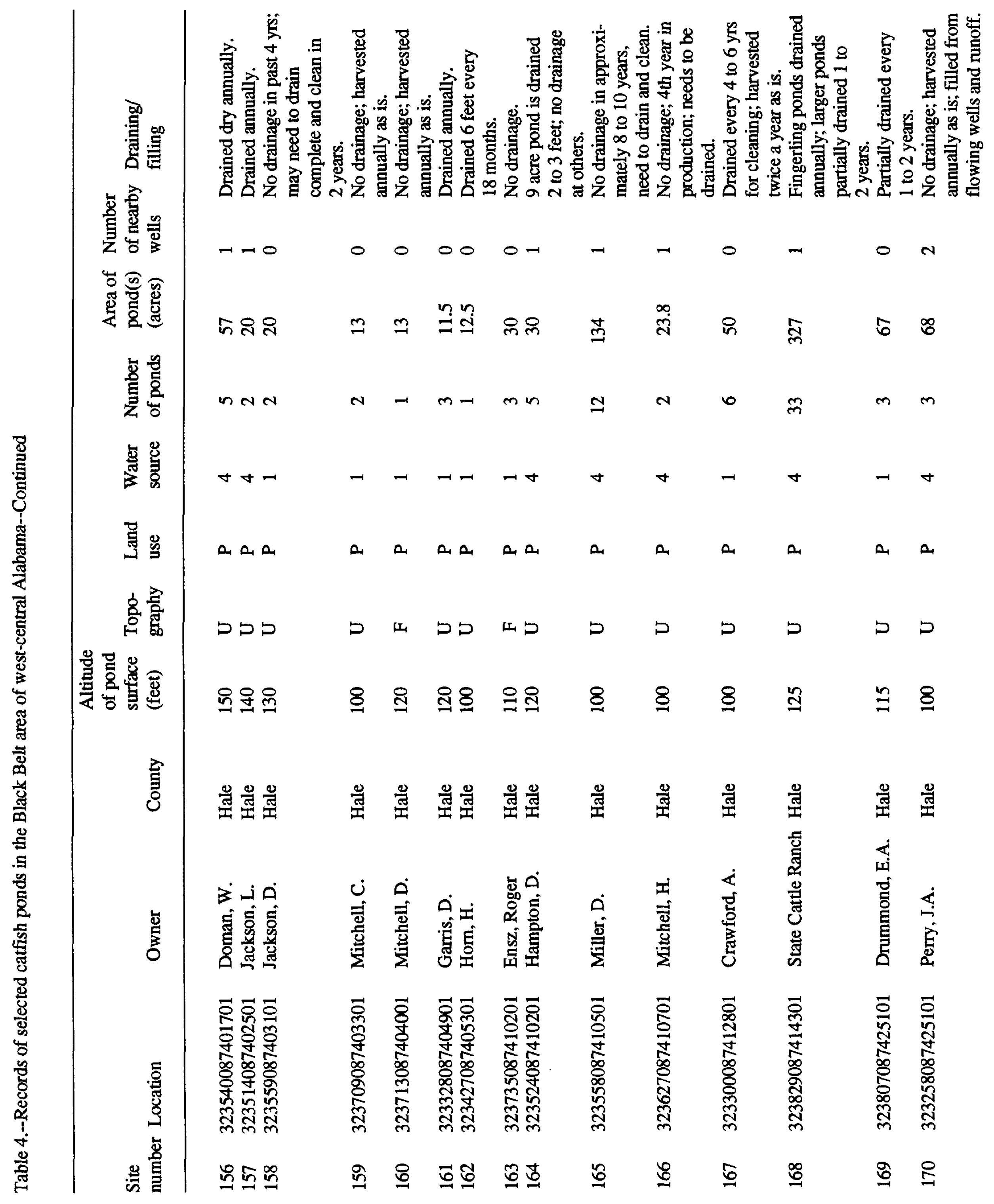




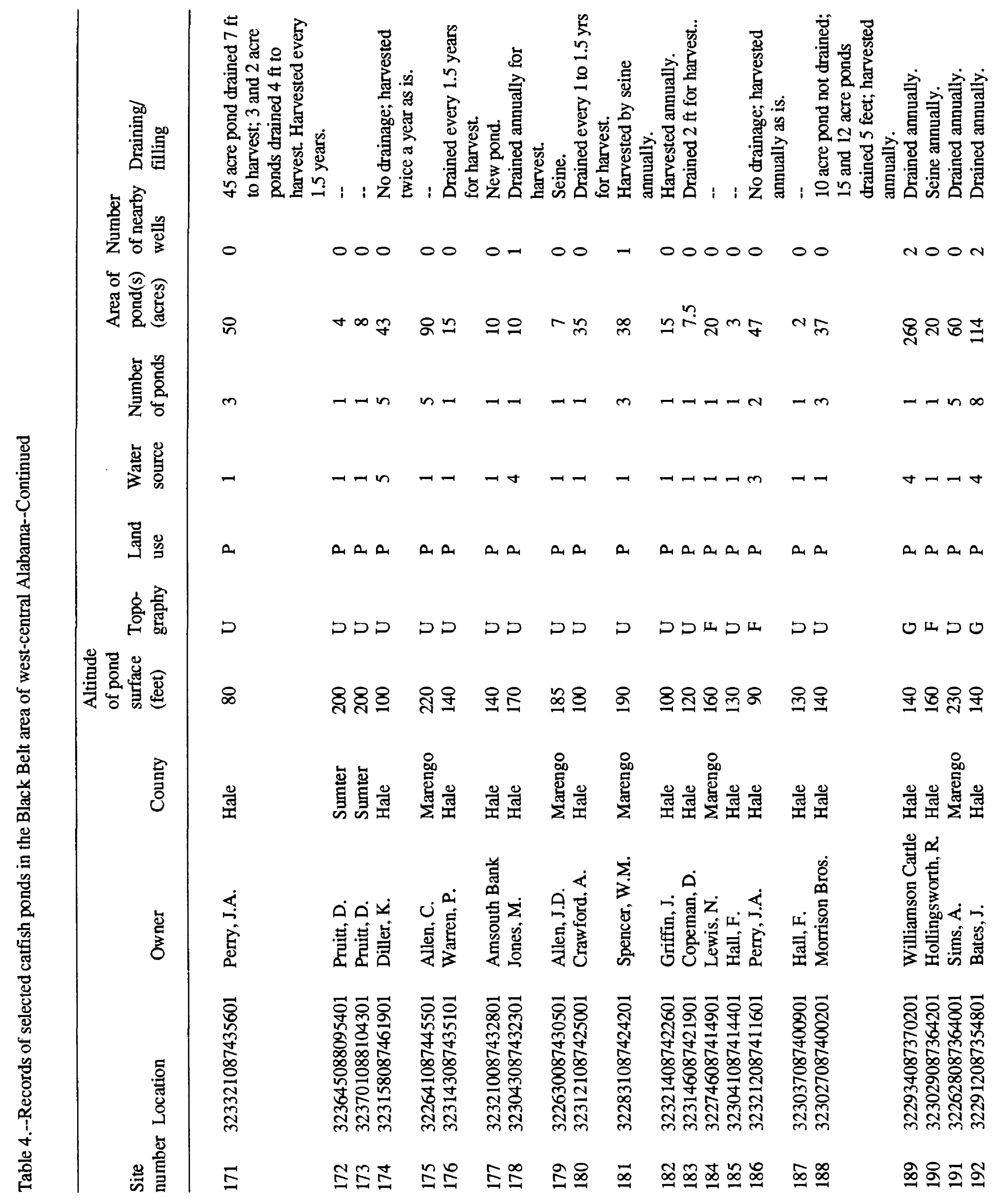




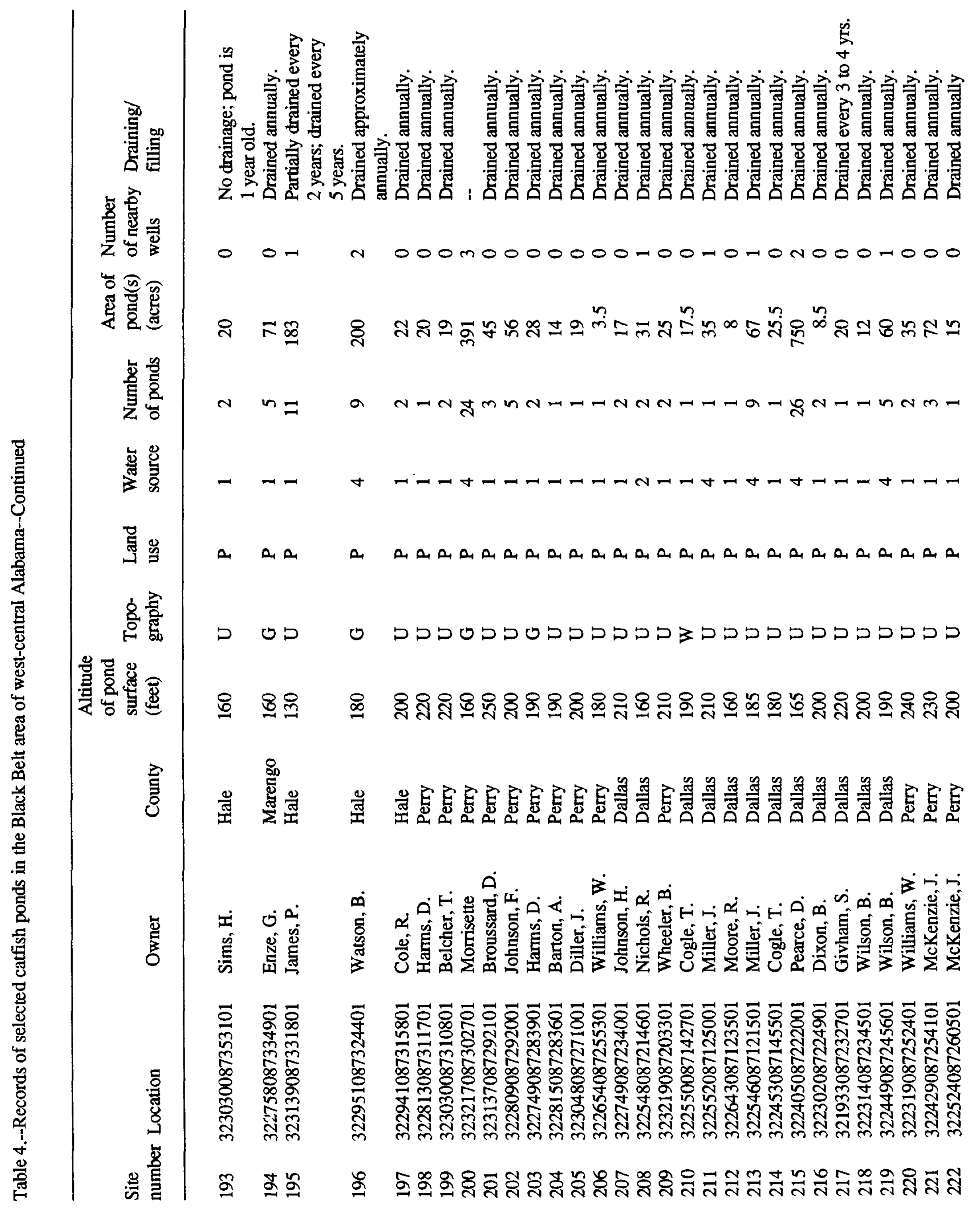




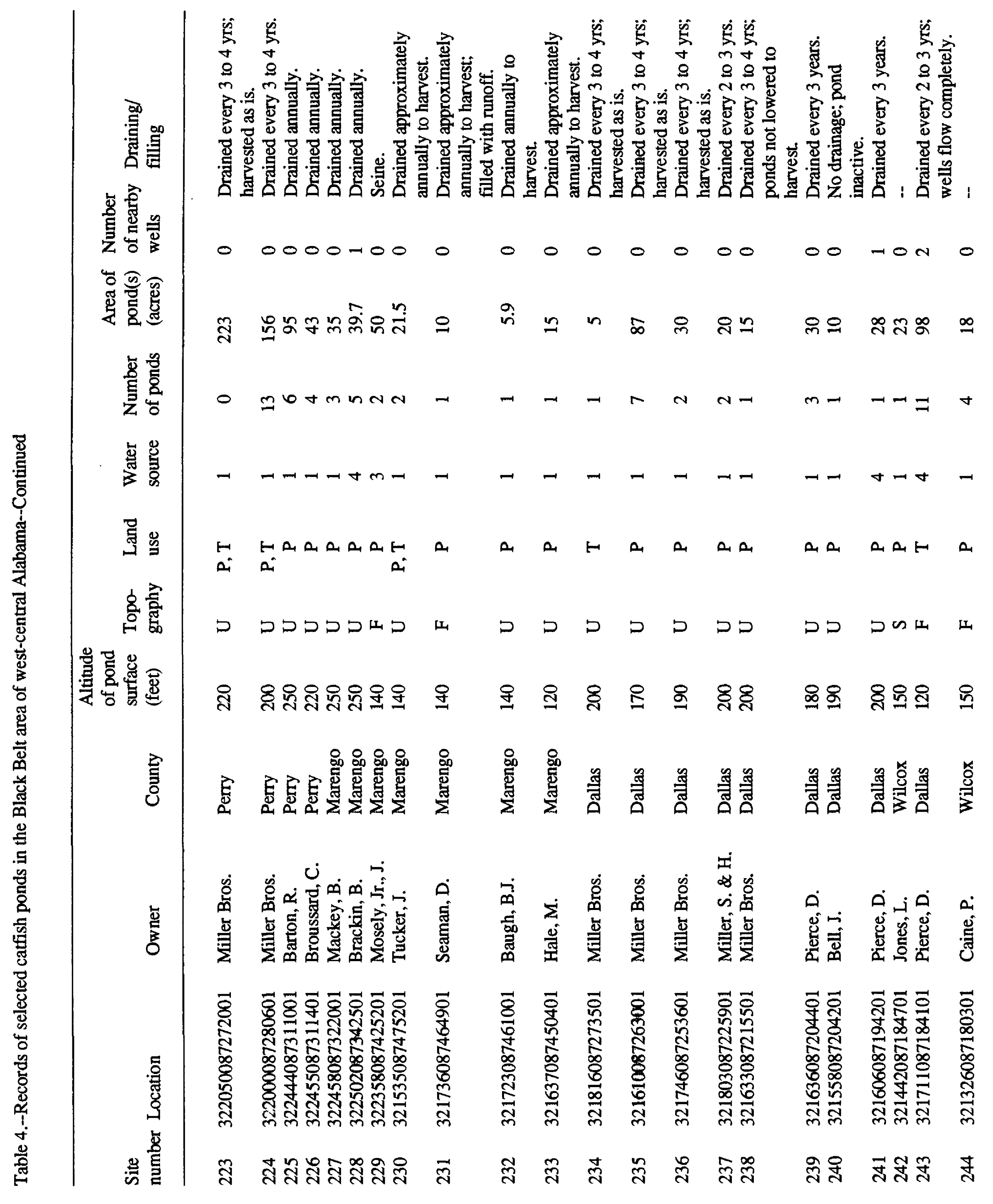




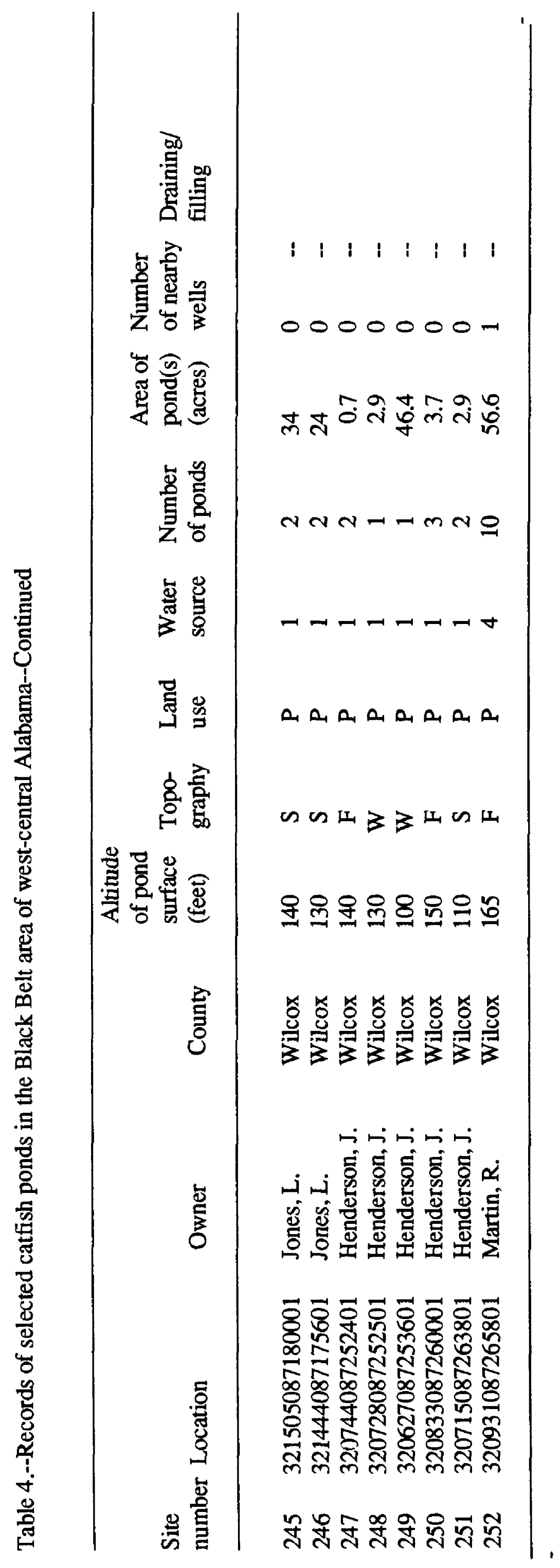


The following assumptions were made when estimating water use for sites that did not have specific water-use data available:

1. A cycle of one drain and fill per year was assumed for ponds without drainage and fill records. Ponds that were reported to be lowered only for harvest were assumed to be lowered 4 feet. A depth of 8 feet was assumed for ponds without depth information.

2. Ponds filled primarily by rainfall runoff, but with some ground water contribution during droughts or other emergencies, were assumed to use 98 percent runoff and 2 percent ground water.

3. Ponds filled primarily with ground water, but with some runoff contribution, were assumed to use 75 percent ground water and 25 percent runoff.

4. Ponds filled with runoff supplemented with either surface water or ground water were assumed to use 75 percent runoff. The remaining 25 percent was from either surface water or ground water.

5. Ponds filled with runoff, ground water, and surface water in undesignated amounts were assumed to use 50 percent runoff, 25 percent ground water, and 25 percent surface water withdrawn from a stream or other water body.

6. Ponds filled with surface water and ground water in undesignated amounts were assumed to use 50 percent surface water and 50 percent ground water.

Estimates of ground water and surface water used to replace evaporation losses from ponds were calculated by multiplying the surface area of the ponds by 10.17 inches which was the sum of rainfall deficit for May through September 1990 when adjusted pan evaporation exceeded rainfall. A pan coefficient of 0.7 was used to estimate water surface evaporation from the class-A land pan evaporation data for the National Oceanic and Atmospheric Administration (NOAA) site at Demopolis (NOAA, 1991; Veihmeyer, 1964). The amounts of ground water and surface water used for operations and evaporation are listed in table 5.

Total water use for aquaculture in the study area in 1990 was $21.83 \mathrm{Mgal} / \mathrm{d} ; 16.08 \mathrm{Mgal} / \mathrm{d}$ from ground-water sources and $5.75 \mathrm{Mgal} / \mathrm{d}$ from surface-water sources (table 5). Pond operations (filling) accounted for $10.01 \mathrm{Mgal} / \mathrm{d}$ of ground water and $3.53 \mathrm{Mgal} / \mathrm{d}$ of surface water. Replacement of evaporation losses accounted for $6.07 \mathrm{Mgal} / \mathrm{d}$ ground water and $2.22 \mathrm{Mgal} / \mathrm{d}$ surface water. Most of the ground water used was from the Eutaw aquifer. 


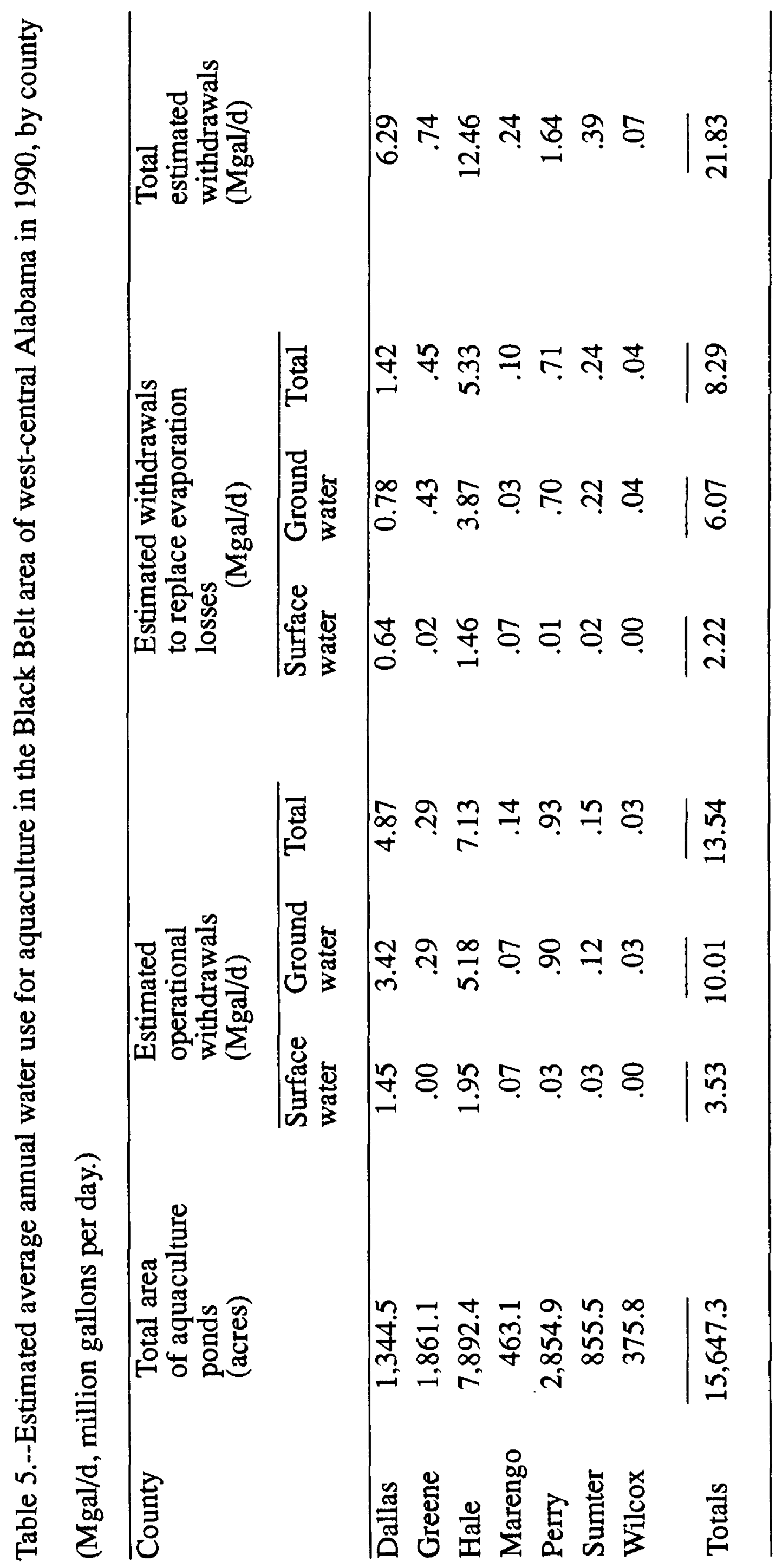




\section{SUMMARY}

Commercial production of catfish in west-central Alabama began about 1970 and by 1990 cattish ponds in the part of the State known as the Black Belt area covered about 16,000 acres. The rapid increase in the number of catfish ponds and the associated demand for ground water prompted State and Federal agencies to initiate a study to define the ground-water resources and water requirements for aquaculture in the area.

Geologic units that crop out in the study area are of sedimentary origin and range in age from Late Cretaceous to Quaternary. The major aquifers in the study area are sand and gravel beds in the Eutaw, Gordo, and Coker Formations. Recharge areas for the major aquifers generally coincide with the outcrops of the Eutaw, Gordo, and Coker Formations along and north of the northern boundary of the study area. The average recharge to the major aquifers, estimated from baseflow analysis, is $11.4 \mathrm{in} / \mathrm{yr}$ in the outcrop areas.

Water from the major aquifers generally is of good quality and suitable for most uses, except in central Greene County and in downdip areas in Marengo and Wilcox Counties where water from the Eutaw aquifer has chloride concentrations in excess of $500 \mathrm{mg} / \mathrm{L}$. Water not suitable for human consumption because of elevated chloride concentrations is used for catfish farming in some parts of the study area.

The potentiometric surfaces of the Eutaw, Gordo, and Coker aquifers have steadily declined since the 1950's in some parts of the study area as a result of ground-water withdrawals for municipal supplies and aquaculture. Local depressions in the potentiometric surface of the major aquifers near large pumping centers have been documented by several investigations.

The total water use for aquaculture in the study area in 1990 was estimated to be 21.83 $\mathrm{Mgal} / \mathrm{d} ; 16.08 \mathrm{Mgal} / \mathrm{d}$ from ground-water sources and $5.75 \mathrm{Mgal} / \mathrm{d}$ from surface-water sources. Pond operations accounted for $13.54 \mathrm{Mgal} / \mathrm{d}$ ground water and $3.53 \mathrm{Mgal} / \mathrm{d}$ surface water. Replacement of evaporation losses accounted for $6.07 \mathrm{Mgal} / \mathrm{g}$ ground water and $2.22 \mathrm{Mgal} / \mathrm{d}$ surface water. 


\section{SELECTED REFERENCES}

American Society of Civil Engineers, 1987, Ground water management (3d ed.): American Society of Civil Engineers Manuals and Reports on Engineering Practice no. 40, 263 p. Baker, R.M., and Mooty, W.S., 1993, Use of water in Alabama, 1990: Geological Survey of Alabama Information Series 59E, $49 \mathrm{p}$.

Barksdale, H.C., 1976, The Eutaw aquifer in Barksdale, H.C. and others, Water content and potential yield of significant aquifers in Alabama: Geological Survey of Alabama open-file report, p. 8.2-8.22.

Causey, L.V., Wilmon, J.R., and Ellard, J.S., 1978, Water availability in Bibb County, Alabama: Geological Survey of Alabama Map 144, 16 p.

Copeland, C.W., 1968, Geology of the Alabama Coastal Plain--Guidebook for 60th annual meeting of American State Geologists, May 6-8: Geological Survey of Alabama Circular 47, $97 \mathrm{p}$.

Davis, M.E., 1980, Ground-water levels in Alabama for observation wells measured periodically August 1952 through July 1977: Geological Survey of Alabama Circular 105, 74 p. 1987, Stratigraphic and hydrogeologic framework of the Alabama Coastal Plain: U.S. Geological Survey Water-Resources Investigations Report 87-4112, 39 p.

Davis, M.E., Sanford, R.H., Jr., and Jefferson, P.O., 1975, Water availability and geology of Hale County, Alabama: Geological Survey of Alabama Map 136, 51 p. 1980, Water availability and geology of Sumter County, Alabama: Geological Survey of Alabama Map 158, 69 p.

DeJarnette, S.S., and Crownover, J.E., 1987, Geohydrology and susceptibility of major aquifers to surface contamination in Alabama; area 6: U.S. Geological Survey Water-Resources Investigations Report 87-4113, 27 p.

Drennen, C.W., 1953, Reclassification of the outcropping Tuscaloosa Group in Alabama: American Association of Petroleum Geologists Bulletin v. 37, no. 3, p. 522-538.

Faye, R.E., and Mayer, G.C., 1990, Ground-water flow and stream-aquifer relations in the northern coastal plain of Georgia and adjacent parts of Alabama and South Carolina: U.S. Geological Survey Water-Resources Investigations Report 88-4143, 82 p.

Fenneman, N.M., 1938, Physiography of Eastern United States: McGraw-Hill Book Co., New York714 p.

Gardner, R.A., 1981, Model of the ground-water flow system of the Gordo and Eutaw aquifers in west-central Alabama: Geological Survey of Alabama Bulletin 118, $30 \mathrm{p}$. 
Kidd, R.E., 1976, Tuscaloosa group aquifers in Barksdale, H.C. and others, Water content and potential yield of significant aquifers in Alabama: Geological Survey of Alabama open-file report, $449 \mathrm{p}$.

Knisel, W.G., and Sheridan, J.M., 1983, Procedure for characterizing hydrologic processes in the coastal plain of the southeastern United States, in Proceedings of the UNESCO International Program on Hydrology on Large Flatlands, Buenos Aires, Argentina, v. 1, p. 191-211.

LaMoreaux, P.E., and others, 1969, Mineral, water, and energy resources of Wilcox County, Alabama: Geological Survey of Alabama Information Series 40, 49 p.

Lamoreaux, P.E., and Toulmin, L.D., 1959, Geology and ground-water resources of Wilcox County, Alabama: Geological Survey of Alabama County Report 4, 280 p.

Linsley, R.K., Jr., Kohler, M.A., and Paulhus, J.L.H., 1982, Hydrology for engineers (3d ed.): McGraw-Hill, New York, 508 p.

Lyne, V., and Hollick, M., 1979, Stochastic time-variable rainfall-runoff modeling, in Hydrology and Water Resources Symposium, Perth, 1979, Proceedings: National Committee on Hydrology and Water Resources of the Institution of Engineers, Australia, p. 89-92.

Meyboom, P., 1961, Estimating ground-water recharge from stream hydrographs: Journal of Geophysical Research, v. 66, no. 4, p. 1203-1214.

Miller, J.A., 1992, Summary of the hydrology of the Southeastern Coastal Plain aquifer system in Mississippi, Alabama, Georgia, and South Carolina: U.S. Geological Survey Professional Paper 1410-A, $38 \mathrm{p}$.

Miller, J.D., Jr., and Causey, L.V., 1958, Geology and ground-water resources of Tuscaloosa County, Alabama, an interim report: Geological Survey of Alabama Information Series 14, $71 \mathrm{p}$.

Monroe, W.H., 1941, Notes on deposits of Selma and Ripley age in Alabama: Geological Survey of Alabama Bulletin 48, $150 \mathrm{p}$.

Mooty, W.S., 1987, Geohydrology and susceptibility of major aquifers to surface contamination in Alabama; area 7: U.S. Geological Survey Water-Resources Investigations Report 87-4109, 28 p.

National Oceanic and Atmospheric Administration, 1991, Alabama precipitation yearly normals 1951-90: Birmingham, River Forecast Office.

Newton, J.G., McCain, J.F., and Knight, A.L., 1971, Water availability of Marengo County, Alabama: Geological Survey of Alabama Map 98, 21 p.

Newton, J.G., Sutcliffe, Horace, Jr., and LaMoreaux, P.E., 1961, Geology and ground-water resources of Marengo County, Alabama: Geological Survey of Alabama County Report 5, 443 p. 
Olmsted, F.H., and Hely, A.G., 1962, Relation between ground water and surface water in Brandywine Creek basin, Pennsylvania: U.S. Geological Survey Professional Paper 417-A, $21 \mathrm{p}$.

Paulson, Q.F., Miller, J.D., Jr., and Drennen, C.W., 1962, Ground-water resources and geology of Tuscaloosa County, Alabama: Geological Survey of Alabama County Report 6, 97 p.

Pettyjohn, W.A., and Henning, Roger, 1979, Preliminary estimate of ground-water recharge rates, related streamflow and water quality in Ohio: Ohio State University Water Resources Center, Project Completion Report no. 552, Columbus, Ohio, 323 p.

Planert, Michael, and Sparkes, S.S., 1985, Estimation of vertical hydraulic conductivity of the clay layer between the Eutaw and Gordo aquifers in the vicinity of Faunsdale, Marengo County, Alabama: U.S. Geological Survey Water-Resources Investigations Report 85-4083, $23 \mathrm{p}$.

Planert, Michael, Williams, J.S., and DeJarnette, S.S., 1991, Geohydrology of the Southeastern Coastal Plain aquifer system in Alabama: U.S. Geological Survey Professional Paper 1410-H, $74 \mathrm{p}$.

Reed, P.C., 1972, Geology of Perry County, Alabama: Geological Survey of Alabama Map 118, $13 \mathrm{p}$.

Reed, P.C., Willmon, J.R., and Jefferson, P.O., 1972, Water availability in Perry County, Alabama: Geological Survey of Alabama Map 127, 42 p.

Rorabaugh, M.I., 1964, Estimating changes in bank storage and ground-water contribution to streamflow: International Association of Scientific Hydrology, Publication no. 63, p. 432-441.

Rutledge, A.T., 1991, A new method for calculating a mathematical expression for streamflow recession, in Ritter, W.F., ed., Irrigation and drainage: National Conference of Irrigation and Drainage Division of the American Society of Civil Engineers, Honolulu, Hawaii, 1991, Proceedings, p. 337-343.

1992, Methods of using streamflow records for estimating total and effective recharge in the Appalachian Valley and Ridge, Piedmont and Blue Ridge physiographic provinces, in Hotchkiss, W.R., and Johnson, A.I., eds., Regional Aquifer Systems of the United States, Aquifers of the southern and eastern States: 27th Annual Conference of American Water Resources Association, New Orleans, Louisiana, 1991, AWRA Monograph Series no. 17, p. 59-73.

Sapp, C.D., and Emplaincourt, Jacques, 1975, Physiographic regions of Alabama: Geological Survey of Alabama Special Map 168.

Scott, J.C., Golden, H.C., and Newton, J.G., 1981, Geology and water availability of Dallas County, Alabama: Geological Survey of Alabama Map 180, 94 p. 
Shirmohammadi, Adel, Knisel, W.G., and Sheridan, J.M., 1984, An approximate method for partitioning daily streamflow data: Journal of Hydrology, v. 74, p. 335-354.

Shirmohammadi, Adel, Sheridan, J.M., and Knisel, W.G., 1987, Regional application of an approximate streamflow partitioning method: Water Resources Bulletin, v. 23, p. 103-111.

Smith, E.A., 1907, The underground water resources of Alabama: Geological Survey of Alabama Monograph 6, $338 \mathrm{p}$.

Toumey, Michael, 1858, The geology of Alabama: Geological Survey of Alabama, 2nd Biennial Report, 292 p.

U.S. Department of Commerce, 1970-90, Climatological data for Alabama: National Oceanic and Atmospheric Climatological Data Reports (published monthly).

Vecchioli, John, Tibbals, C.H., Duen, A.D., and Hutchinson, C.B., 1990, Ground-water recharge in Florida--A pilot study in Okaloosa, Pasco, and Volusia Counties: U.S. Geological Survey Water-Resources Investigations Report 90-4195, $16 \mathrm{p}$.

Veihmeyer, F.J., 1964, Evapotranspiration in Chow, V.T. (editor), Handbook of applied hydrology: McGraw-Hill, New York, p. 11.1-11.38.

Wahl, K.D., 1965, Ground-water resources of Pickens County, Alabama: Geological Survey of Alabama Bulletin 83, 84 p. 1966, Geology and ground-water resources of Greene County, Alabama: Geological Survey of Alabama Bulletin 86, $93 \mathrm{p}$.

Williams, J.S., DeJarnette, S.S., and Planert, Michael, 1986a, Potentiometric-surface and wateruse map of the Tuscaloosa aquifer in Alabama, fall 1982: U.S. Geological Survey WaterResources Investigations Report 85-4174, 1 sheet. 1986b, Potentiometric-surface, ground-water withdrawals and recharge areas in the Eutaw aquifer in Alabama, fall 1982: U.S. Geological Survey Water-Resources Investigations Report 86-4121, 1 sheet.

Williams, J.S., Planert, Michael, DeJarnette, S.S., 1986, Potentiometric-surface, ground-water withdrawals and recharge areas for the Providence-Ripley aquifer in Alabama, fall 1982: U.S. Geological Survey Water-Resources Investigations Report 86-4118, 1 sheet. 